\title{
Self-Similar Force-Free Wind From an Accretion Disk
}

\author{
Ramesh Narayan ${ }^{1 \star}$, Jonathan C. McKinney $^{1 \star}$, and Alison J. Farmer ${ }^{1 \star}$ \\ ${ }^{1}$ Institute for Theory and Computation, Center for Astrophysics, Harvard University, 60 Garden St., Cambridge, MA, 02138
}

Accepted 2006 Oct 26. Received 2006 Sept 16; in original form 2006 Sept 16

\begin{abstract}
We consider a self-similar force-free wind flowing out of an infinitely thin disk located in the equatorial plane. On the disk plane, we assume that the magnetic stream function $P$ scales as $P \propto R^{\nu}$, where $R$ is the cylindrical radius. We also assume that the azimuthal velocity in the disk is constant: $v_{\phi}=M c$, where $M<1$ is a constant. For each choice of the parameters $\nu$ and $M$, we find an infinite number of solutions that are physically well-behaved and have fluid velocity $\leqslant c$ throughout the domain of interest. Among these solutions, we show via physical arguments and time-dependent numerical simulations that the minimum-torque solution, i.e., the solution with the smallest amount of toroidal field, is the one picked by a real system. For $\nu \geqslant 1$, the Lorentz factor of the outflow increases along a field line as $\gamma \approx M\left(z / R_{\mathrm{fp}}\right)^{(2-\nu) / 2} \approx R / R_{\mathrm{A}}$, where $R_{\mathrm{fp}}$ is the radius of the foot-point of the field line on the disk and $R_{\mathrm{A}}=R_{\mathrm{fp}} / M$ is the cylindrical radius at which the field line crosses the Alfven surface or the light cylinder. For $\nu<1$, the Lorentz factor follows the same scaling for $z / R_{\mathrm{fp}}<M^{-1 /(1-\nu)}$, but at larger distances it grows more slowly: $\gamma \approx\left(z / R_{\mathrm{fp}}\right)^{\nu / 2}$. For either regime of $\nu$, the dependence of $\gamma$ on $M$ shows that the rotation of the disk plays a strong role in jet acceleration. On the other hand, the poloidal shape of a field line is given by $z / R_{\mathrm{fp}} \approx$ $\left(R / R_{\mathrm{fp}}\right)^{2 /(2-\nu)}$ and is independent of $M$. Thus rotation has neither a collimating nor a decollimating effect on field lines, suggesting that relativistic astrophysical jets are not collimated by the rotational winding up of the magnetic field.
\end{abstract}

Key words: accretion disks, black hole physics, galaxies: jets

\section{INTRODUCTION}

Although relativistic jets from accreting black holes have been known and have been studied for decades, the physics behind the production, acceleration and collimation of these jets is still poorly understood. Broadly, there are two schools of thought on the launching of jets. According to the first (e.g., Lovelace 1976), jets are the innermost and most energetic region of an extended outflow from the black hole accretion disk. The power for the jet thus comes from the disk. In the other view, the jet is powered by the free energy associated with the spin of the central black hole (Blandford \& Znajek 1977). The jet phenomenon is then a remarkable manifestation of the Penrose (1969) process.

The fact that jets from black holes are observed to accelerate to relativistic velocities, despite the absence of any significant radiative or thermal driving, strongly suggests that magnetic fields play a dynamically important role. This motivates the study of magnetohydrodynamic (MHD) mod-

\footnotetext{
* E-mail: narayan@cfa.harvard.edu (RN) ; jmckinney@cfa.harvard.edu (JCM); afarmer@cfa.harvard.edu (AJF)
}

els of jets. Moreover, given the difficulty of achieving selfcollimation in an isolated jet - e.g., Michel's (1973a) solution for a spinning monopole has no collimation at all (see $\S 3.2$ below) - it is reasonable to suppose that the presence of an extended outflow surrounding the jet is needed for maintaining both the collimation and dynamical stability of the jet, as stressed for instance by Appl \& Camenzind (1992, 1993) and Beskin \& Malyshkin (2000). This external flow is presumably a wind of some sort from the disk. It is thus of interest to study the dynamics of extended, nearly self-similar, MHD, disk outflows.

Given the complexity of the full relativistic MHD equations, a variety of simplifications have been introduced by different authors. Many studies make a nonrelativistic approximation (e.g., Blandford \& Payne 1982; Heyvaerts \& Norman 1989; Contopoulos \& Lovelace 1994; Contopoulos 1995b; Ostriker 1997). Although it is adequate for certain aspects of the problem, this approach fails to capture the presence of a light cylinder (or relativistic Alfven surface), an explicitly relativistic phenomenon that likely plays an important role in jet physics. The reader is referred to the seminal work on light cylinders by Goldreich \& Julian (1969) who 
discussed pulsar magnetospheres and Okamoto $(1974,1978)$ who considered disk winds, as well as the work of Lovelace et al. (1986), Nitta, Takahashi \& Tomimatsu (1991), Beskin \& Pariev (1993) and Beskin (1997) who included the effects of general relativity.

In considering relativistic winds, one could either work with the full MHD equations (e.g., Vlahakis \& Konigl 2003), or make further simplifications. One possibility is to assume that the outflowing gas is cold (e.g., Okamoto 1978; Li et al. 1992; Contopoulos 1994) so that only the inertia of the fluid contributes to the dynamics and there are no effects due to pressure or internal energy. An even more drastic simplification is to ignore gas inertia as well. In this force-free approximation, the plasma supplies charges and currents as needed to support the electromagnetic fields, but it has no other dynamical role.

Force-free electrodynamics is the simplest and cleanest model of magnetized relativistic outflows. If magnetic fields are at all important in disk winds and jets, one might hope that the force-free approximation would capture a good fraction of the relevant physics. Early discussions of forcefree disk winds may be found in Okamoto (1974), Blandford (1976) and Blandford \& Znajek (1977). Recently, force-free electrodynamics has been formulated as a system of timedependent equations (Komissarov 2002b, 2004; McKinnev 2006a; Spitkovskv 2006). These time-dependent force-free equations have been used to study black hole and neutron star magnetospheres (Komissarov 2001, 2002a b, 2004, 2006; McKinnev 2006a b; McKinnev \& Naravan 2006b; Spitkovskv 2006).

Force-free models have gained new relevance as a result of time-dependent general-relativistic MHD simulations of black hole accretion flows (e.g., McKinney \& Gammie 2004; Gammie et al. 2004; de Villiers et al. 2005). These simulations reveal a nearly force-free highly relativistic jet forming spontaneously along the symmetry axis and accelerating rapidly outwards. Moreover, the height-integrated azimuthal current within the turbulent disk is found to have a surprisingly simple power-law behavior (McKinney \& Narayan $2006 \mathrm{a}, \mathrm{b}), d I_{\phi} / d R \propto R^{-5 / 4}$, where $R$ is the radius and $I_{\phi}(R)$ is the azimuthal current enclosed inside $R$. In addition, an idealized force-free model with an equatorial rotating current sheet with azimuthal current varying as $R^{-5 / 4}$, which is equivalent to the magnetic stream function varying as $P \propto R^{3 / 4}$ (or $\nu=3 / 4$ in eq. 14 below), gives a surprisingly good match to a variety of properties of the simulated jet such as the poloidal configuration of the magnetic field, the collimation angle of the jet, the Lorentz factor, etc. Thus, it appears that simple disk wind models with self-similar (power-law) scalings may capture key aspects of the jet problem. We should note, however, that self-similar disk wind models with the particular scaling $P \propto R^{3 / 4}$ have a checkered history and there has been some confusion on whether or not well-behaved solutions that extend to large distances from the disk are present at all. Blandford \& Payne (1982) and Contopoulos (1995a), for instance, both find that such solutions do not exist.

In this paper we analyze the structure of self-similar force-free disk winds for a range of power-law profiles of the stream function, including the case $P \propto R^{3 / 4} . \S 2$ introduces our model, which is closely similar to the model of Contopou$\operatorname{los}(1995 \mathrm{a})$ and is a self-similar specialization of Okamoto's
(1974) more general analysis. We show that the problem is reduced to solving the second-order differential equation (26) with appropriate boundary conditions. $\S 3$ discusses a number of analytical results and maps out the different kinds of solutions that are possible. $\S 4$ presents results from a numerical analysis of the basic differential equation and identifies regions in parameter space where the different solution types are found. $\S 5$ presents time-dependent numerical simulations of force-free disk winds using a relativistic force-free code and draws some general conclusions as to which of the many solutions are relevant for given situations. The paper concludes with a discussion in $\S 6$. The Appendix discusses some mathematical properties of the differential equation (26).

\section{THE MODEL}

\subsection{Okamoto's (1974) Force-Free Field Equation}

We consider a steady, axisymmetric force-free field in cylindrical coordinates: $R, \phi, z$. Since $\vec{\nabla} \cdot \vec{B}=0$, the magnetic field $\vec{B}$ can be written in terms of a vector potential: $\vec{B}=\vec{\nabla} \times \vec{A}$. Furthermore, since we have assumed axisymmetry $(\partial / \partial \phi=0)$, the poloidal component $\overrightarrow{B_{p}}$ of the magnetic field (the projection on the $R z$ plane) can depend only on the $\phi$ component $A_{\phi}$ of the vector potential. Thus, we may write the field quite generally as

$$
\begin{aligned}
\vec{B} & =\overrightarrow{B_{p}}+B_{\phi} \hat{\phi}=\vec{\nabla} \times\left(A_{\phi} \hat{\phi}\right)+B_{\phi} \hat{\phi} \\
& =\left[-\frac{1}{R} \frac{\partial\left(R A_{\phi}\right)}{\partial z}, B_{\phi}, \frac{1}{R} \frac{\partial\left(R A_{\phi}\right)}{\partial R}\right],
\end{aligned}
$$

where $B_{\phi}$ is the toroidal component of the field. It is standard to rewrite this in terms of the stream function $P \equiv$ $R A_{\phi}$ (the stream function is also often represented by the symbol $\psi)$ :

$\vec{B}=\left[-\frac{1}{R} \frac{\partial P}{\partial z}, B_{\phi}, \frac{1}{R} \frac{\partial P}{\partial R}\right]$.

The magnetic flux enclosed within radius $R$ is given by

$\Phi(R)=\int_{0}^{R} B_{z} 2 \pi R^{\prime} d R^{\prime}=2 \pi P(R)$.

Thus, apart from a factor of $2 \pi$, the stream function $P$ is the same as the enclosed magnetic flux.

Since the enclosed flux interior to a given field line is constant, independent of the $z$ at which it is measured, it is clear that $P$ must be a constant along each field line. This can also be shown explicitly from equation (2) by verifying that $\vec{\nabla} P \cdot \vec{B}=0$. Thus, each field line is uniquely specified by its value of $P$. Another quantity that is constant on each field line is the angular velocity $\Omega$. Since we imagine that the field line is embedded in an accretion disk and corotates with it at its foot-point, the angular velocity of the entire field line out to infinity is determined by the rotation of the disk at the foot-point. $\Omega$ is clearly a function only of $P$.

By definition, in the frame rotating with the angular velocity $\Omega$ of a field line we are in the comoving frame of the fluid and hence there is no electric field (because we assume infinite conductivity). Therefore, back in the nonrotating frame, there must be an electric field, which is given by

$\vec{E}=-\frac{\Omega R}{c} \hat{\phi} \times \vec{B}=\frac{\Omega R}{c}\left(-B_{z}, 0, B_{R}\right)=-\frac{\Omega}{c} \vec{\nabla} P$. 
The electric charge density may be obtained from Gauss's law:

$$
\begin{aligned}
\rho_{e} & =\frac{1}{4 \pi} \vec{\nabla} \cdot \vec{E} \\
& =-\frac{\Omega}{4 \pi c}\left(\frac{\partial^{2} P}{\partial R^{2}}+\frac{1}{R} \frac{\partial P}{\partial R}+\frac{\partial^{2} P}{\partial z^{2}}\right)-\frac{1}{4 \pi c}|\nabla P|^{2} \frac{d \Omega}{d P}
\end{aligned}
$$

In steady state the current density is given by

$\vec{j} \equiv \overrightarrow{j_{p}}+j_{\phi} \hat{\phi}=\frac{c}{4 \pi} \vec{\nabla} \times \vec{B}$,

where we have once again decomposed the vector into poloidal and toroidal components. This gives

$$
\begin{aligned}
& j_{\phi}=\frac{c}{4 \pi}(\vec{\nabla} \times \vec{B})_{\phi}=-\frac{c}{4 \pi R}\left(\frac{\partial^{2} P}{\partial R^{2}}-\frac{1}{R} \frac{\partial P}{\partial R}+\frac{\partial^{2} P}{\partial z^{2}}\right) \\
& \overrightarrow{j_{p}}=\frac{c}{4 \pi}(\vec{\nabla} \times \vec{B})_{p}=-\frac{c}{4 \pi} \frac{\partial B_{\phi}}{\partial z} \hat{R}+\frac{c}{4 \pi R} \frac{\partial\left(R B_{\phi}\right)}{\partial R} \hat{z}
\end{aligned}
$$

In the force-free limit we have no inertial terms in the momentum equation, so the sum of all the electromagnetic forces at each point must be zero. Thus the equation of motion takes the simple form

$\rho_{e} \vec{E}+\frac{1}{c} \vec{j} \times \vec{B}=0$.

Since $\vec{E}$ has no toroidal component, neither can $\vec{j} \times \vec{B}$. Thus $\overrightarrow{j_{p}}$ and $\overrightarrow{B_{p}}$ have to be parallel to each other. Comparing the expression for $\overrightarrow{j_{p}}$ in equation (8) with that for $\vec{B}$ in equation (2), we see that the quantity $R B_{\phi}$ must be a unique function of $P$. Hence this is a third quantity that is constant along a field line:

$$
\begin{aligned}
R B_{\phi} & =\beta(P), \\
\overrightarrow{j_{p}} & =\frac{c}{4 \pi} \frac{d \beta}{d P} \overrightarrow{B_{p}} .
\end{aligned}
$$

Note that, at each point, $\beta$ is proportional to the net enclosed current in the $z$-direction

Taking the dot product of equation (9) with $\vec{E}$, we obtain

$\rho_{e} E^{2}+\frac{1}{c} \vec{E} \cdot(\vec{j} \times \vec{B})$.

Substituting the expressions written down earlier for the various quantities, and after some algebra, we finally obtain the following partial differential equation for $P$ :

$$
\begin{aligned}
0 & =\left(1-\frac{\Omega^{2} R^{2}}{c^{2}}\right) \frac{\partial^{2} P}{\partial R^{2}}-\left(1+\frac{\Omega^{2} R^{2}}{c^{2}}\right) \frac{1}{R} \frac{\partial P}{\partial R} \\
& +\left(1-\frac{\Omega^{2} R^{2}}{c^{2}}\right) \frac{\partial^{2} P}{\partial z^{2}} \beta \frac{d \beta}{d P}-\frac{\Omega R^{2}}{c^{2}} \frac{d \Omega}{d P}|\nabla P|^{2}
\end{aligned}
$$

This is the force-free field equation of Okamoto (1974, compare with his eq. 43; simpler versions of this equation without the last term were discussed earlier by Mestel 1973, Michel 1973b and Scharlemann \& Wagoner 1973).

\subsection{Self-Similar Disk Wind}

The discussion so far was quite general. All we assumed were the following conditions: (i) steady state, (ii) axisymmetry, (iii) force-free (i.e., negligible inertia), (iv) infinite conductivity. Now we explicitly consider the problem at hand, viz., a self-similar force-free wind flowing out of an accretion disk (Contopoulos 1995a). We assume that the disk is infinitely thin and located at the equatorial plane, $z=0$. Each field line is identified by the radius $R_{\mathrm{fp}}$ of its foot-point on the disk. The disk supplies two boundary conditions at the footpoint:

1. The magnetic flux $\Phi\left(R_{\mathrm{fp}}\right)$ enclosed inside radius $R_{\mathrm{fp}}$ : This determines the stream function $P\left(R_{\mathrm{fp}}\right)=\Phi\left(R_{\mathrm{fp}}\right) / 2 \pi$ of each field line as a function of the radius of its footpoint.

2 . The angular velocity $\Omega\left(R_{\mathrm{fp}}\right)$ of the disk at radius $R_{\mathrm{fp}}$ : The foot-point of the field line is dragged around by the disk at the angular velocity $\Omega\left(R_{\mathrm{fp}}\right)$ and since $\Omega$ is constant along each field line this angular velocity is imparted to the entire line out to infinity.

Assuming that we have a self-similar disk with a powerlaw structure, we write the stream function on the disk plane as

$\left.P(R, z)\right|_{z=0} \propto R^{\nu}, \quad 0 \leqslant \nu \leqslant 2$.

By this definition, $\nu$ is equivalent to the index $x$ in Contopoulos (1995a) and the index $F$ in Vlahakis \& Konigl (2003). The vertical component of the magnetic field on the disk plane then scales as

$\left.B_{z}(R, z)\right|_{z=0}=\left(\frac{1}{R} \frac{\partial P}{\partial R}\right)_{z=0} \propto R^{\nu-2}$.

Since we would like the enclosed magnetic flux to vanish as $R \rightarrow 0$, we impose the condition $\nu \geqslant 0$. Also, since we are not interested in solutions in which the magnetic field strength increases with increasing radius, we restrict ourselves to $\nu \leqslant 2$. Note that the monopole solution of Michel (1973a) corresponds to $\nu=0$, the problem considered by Blandford \& Payne (1982) corresponds to $\nu=3 / 4$, the paraboloidal field model of Blandford (1976) corresponds to $\nu=1$, and the models discussed by Ostriker (1997) correspond to the range $1<\nu<2$.

The power-law form of the boundary condition (14) motivates us to consider the following self-similar form for the stream function,

$P(R, z)=R^{\nu} T(u)$,

where the self-similar coordinate $u$ (called $Z$ by Contopoulos 1995a) is given by

$u \equiv \frac{z}{R}$.

For convenience we set

$T(0)=1$,

which is equivalent to absorbing any coefficient on the righthand side of equation (16) into the definition of $R$. Since field lines live on surfaces of constant $P$, a field line going through the point $(R, z)$ has its foot-point at radius

$R_{\mathrm{fp}}=R T^{1 / \nu}(z / R)$.

Equivalently, for a given $R_{\mathrm{fp}}$, the shape of the field line in the poloidal plane is given by the parametric equations

$R(u)=R_{\mathrm{fp}} T^{-1 / \nu}(u), \quad z(u)=R_{\mathrm{fp}} u T^{-1 / \nu}(u)$.

Consider now the angular velocity. From equation (13), it is clear that the dimensionless ratio $\Omega R / c$ plays a prominent role in this model. In order to obtain a self-similar solution, this quantity has to be constant on the disk plane. Thus we write 


$$
\left(\frac{\Omega R}{c}\right)_{z=0}=M=\text { constant }
$$

where $M\left(=1 / c_{n}\right.$ in Contopoulos 1995a) represents a sort of "Mach number" of the angular motion. Note that the azimuthal velocity follows a "flat rotation curve," not a Keplerian profile. This is not the ideal choice for an accretion disk, but it is the only profile that is consistent with selfsimilarity (e.g., Li et al. 1992; Contopoulos 1995a; Vlahakis $\&$ Konigl 2003) ${ }^{1}$. Since $\Omega$ is constant on a field line, the angular velocity at any general $(R, z)$ is given by

$$
\frac{\Omega R}{c}=M T^{-1 / \nu} \text {. }
$$

Consider now the third conserved quantity on a field line, the quantity $\beta$. Under self-similarity, we expect the ratio of the toroidal and poloidal field strengths at the disk plane to be a constant. Thus we assume that

$$
\left(\frac{B_{\phi}}{B_{z}}\right)_{z=0}=-s M=\text { constant. }
$$

We include $M$ on the right-hand side since we expect the amount of toroidal field in the wind to be roughly proportional to the rotation rate of the disk. We also introduce a negative sign because we expect field lines to be swept back with respect to the direction of rotation. (The quantity $H_{0}$ in Contopoulos 1995a is $-s M$ in our notation.) We then obtain

$$
\begin{aligned}
\beta & =-\nu s M R^{\nu-1} T^{(\nu-1) / \nu} \\
\beta \frac{d \beta}{d P} & =\nu(\nu-1) s^{2} M^{2} R^{\nu-2} T^{(\nu-2) / \nu} .
\end{aligned}
$$

Substituting the various self-similar scalings written down above into equation (13), we finally obtain the differential equation satisfied by the similarity function $T(u)$ :

$$
\begin{aligned}
& 0=\left(u^{2}+1\right) T^{\prime \prime}+(3-2 \nu) u T^{\prime}+\nu(\nu-2) T \\
&-\quad M^{2} T^{-(\nu+2) / \nu}\left[\left(u^{2}+1\right) T T^{\prime \prime}-\left(u^{2}+1\right) T^{\prime 2} / \nu\right. \\
&\left.+(3-2 \nu) u T T^{\prime}+\nu(\nu-1)\left(1-s^{2}\right) T^{2}\right]
\end{aligned}
$$

This is the fundamental equation that we need to solve to study self-similar force-free disk winds. Once we have a solution for $T(u)$, we can calculate all other quantities of interest. For instance, the three components of the magnetic field are given by

$$
\begin{aligned}
B_{R}(R, z) & =-R^{\nu-2} T^{\prime}(u), \\
B_{\phi}(R, z) & =-\nu s M R^{\nu-2} T^{(\nu-1) / \nu}(u), \\
B_{z}(R, z) & =R^{\nu-2}\left[\nu T(u)-u T^{\prime}(u)\right],
\end{aligned}
$$

where as always $u=z / R$.

\footnotetext{
1 This restriction arises because we are working with a relativistic theory in which $c$ introduces a fundamental unit of velocity. Nonrelativistic self-similar models have the freedom to choose any power-law for the radial profile of the angular velocity, including in particular a Keplerian profile, but the price for this is that they ignore all relativistic effects.
}

\subsection{The Alfven Critical Surface}

The highest derivative $T^{\prime \prime}$ in equation (26) is multiplied by a factor of the form

$$
\left(1-M^{2} T^{-2 / \nu}\right) \equiv\left(1-\frac{\Omega^{2} R^{2}}{c^{2}}\right),
$$

and so the differential equation is clearly singular when this factor goes to zero. The singularity corresponds to the Alfven critical surface, also called the light cylinder in some of the earlier literature. It is located at the radius

$R_{\mathrm{A}}=\frac{R_{\mathrm{fp}}}{M}$.

Note that the Alfven surface occurs at a specific value of $u=u_{c}$ and has the shape of a cone. This is in contrast to the pulsar magnetosphere problem where it occurs at a single radius, thereby motivating the terminology "light cylinder" in that case.

When $u=u_{c}$ the factor given in eq (30) goes to zero, and therefore in order for the solution to be well-behaved the rest of the terms in (26) that do not involve $T^{\prime \prime}$ should also add up to zero. We thus obtain the following two regularity conditions at $u_{c}$ :

$$
\begin{aligned}
T\left(u_{c}\right) & =M^{\nu}, \\
T^{\prime}\left(u_{c}\right) & =-\nu M^{\nu}\left[\frac{1-(\nu-1) s^{2}}{u_{c}^{2}+1}\right]^{1 / 2} .
\end{aligned}
$$

The negative sign on the second condition is required for a physically sensible solution.

For a given solution $T(u)$, it is easy to determine whether any local stretch of the solution is inside or outside the Alfven surface ; here and elsewhere, we use the term "inside" for $u<u_{c}$ and "outside" for $u>u_{c}$. If $T(u)>T\left(u_{c}\right)=M^{\nu}$, then we are inside the Alfven surface, and if $T(u)<M^{\nu}$ we are outside. Note that, for $\nu<1$, the condition (33) can be satisfied for any value of $s$. However, for $\nu>1$, a solution is possible only if $s^{2} \leqslant 1 /(\nu-1)$. If $|s|$ is larger than this limit, the solution cannot pass through the Alfven surface.

\subsection{Flow Velocity}

While the azimuthal velocity of a field line passing through $(R, z)$ is given by equation (22), this does not determine the fluid velocity. By assumption, the plasma has no inertia, so we are allowed to add any velocity parallel (or antiparallel) to the field line without changing the solution in any way. There is thus considerable ambiguity as to what we mean by the fluid velocity. We could, however, ask what the minimum fluid velocity is, i.e., we could choose the parallel velocity such that the net velocity of the plasma is a minimum. This minimum velocity is the "particle drift velocity,"

$v_{\min } \equiv \frac{|\vec{S}|}{B^{2} / 4 \pi}$,

where

$$
\begin{aligned}
\vec{S} & =\frac{c}{4 \pi} \vec{E} \times \vec{B} \\
& =\frac{\Omega R}{4 \pi}\left[-B_{\phi} B_{R} \hat{R}+\left(B_{R}^{2}+B_{z}^{2}\right) \hat{\phi}-B_{\phi} B_{z} \hat{z}\right]
\end{aligned}
$$


is the Poynting flux, $\vec{E}$ is the electric field given in equation (4), and $B$ is the total magnetic field strength. We then have

$\frac{v_{\mathrm{min}}}{c}=\Omega R \frac{B_{p}}{B}=\frac{R}{R_{\mathrm{LC}}} \frac{B_{p}}{B}=M \frac{R}{R_{\mathrm{fp}}} \frac{B_{p}}{B}$,

where $B_{p}$ is the magnitude of the poloidal component of the magnetic field.

Another relevant speed is the "energy flow speed," which is given by

$v_{E} \equiv \frac{|\vec{S}|}{U}$,

where $U$ is the electromagnetic energy density given by

$U=\frac{1}{8 \pi}\left[|\vec{E}|^{2}+|\vec{B}|^{2}\right]=\frac{B_{p}^{2}}{8 \pi}\left(\frac{\Omega^{2} R^{2}}{c^{2}}+1\right)+\frac{B_{\phi}^{2}}{8 \pi}$.

The energy flow speed is related to the particle drift speed by

$v_{E}=\frac{2 v_{\min }}{1+v_{\min }^{2} / c^{2}}$,

which shows that $v_{E}$ is always $\leqslant c$. Intuitively, one expects a physically sensible solution to have $v_{E}, v_{\min } \rightarrow c$ at large distance from the disk, which is equivalent to the condition that the fast critical surface is at infinity. This requirement is met when the following two conditions are satisfied:

$-\frac{B_{\phi}}{B_{p}} \rightarrow \frac{\Omega R}{c} \rightarrow \infty$.

The second condition says that we have to be infinitely far outside the Alfven surface, i.e., we should focus on the "paraboloidal" solutions discussed in $\S 3$.

So long as we are inside the Alfven surface, we are guaranteed that $v_{\min }<c$. However, once a field line goes outside the Alfven surface $(\Omega R>c)$, there is nothing in the problem to prevent $v_{\min }$ from exceeding $c$. This highlights a major weakness of the force-free model. Even though the model is fully relativistic and causal, it has no way of enforcing $v<c$ for the fluid it is meant to describe because it does not explicitly make use of the inertia. Note that in any region that has $v_{\min }>c$, the electric field strength exceeds the magnetic field strength, allowing unlimited electrostatic acceleration of charged particles.

In this paper, we focus our attention on solutions that have $v_{\min } \leqslant c$ for all $u$ of interest, i.e., all $R$ and $z$ spanned by a given problem. We call such solutions physically viable and consider solutions that violate this condition as "unphysical." The restriction to physical solutions plays the role of a boundary condition on the solution (see $\S$ [2.5). From equation (36) we see that requiring the solution to be physical is equivalent to requiring a minimum amount of toroidal field, or equivalently, a minimum level of enclosed $j_{z}$ (see the comment below eq. 11).

We should note, however, that even solutions we consider unphysical under the above strict criterion may have a role under some circumstances. For instance, an MHD flow with plasma inertia may have a force-free zone that matches the corresponding segment of one of our unphysical solutions but may then deviate into a non-force-free flow in the region where the unphysical solution has $v_{\min }>c$ (e.g., Contopoulos 1995a). A comparison of the force-free solutions discussed in this paper with self-similar MHD solutions would thus be very instructive.

\subsection{Boundary Conditions}

Let us count the number of degrees of freedom in the problem and the boundary conditions we need. Since equation (26) is a second order differential equation, it requires two boundary conditions. The constants $\nu$ and $M$ are clearly determined by the properties of the disk and should be treated as externally supplied parameters of the problem. However, the other two constants, $s$ and $u_{c}$, are not free parameters. They have to be determined self-consistently in the process of solving the problem, i.e., they are eigenvalues.

Consider first a problem in which field lines cross the Alfven surface. To determine the two eigenvalues $s$ and $u_{c}$ we have the two regularity conditions (32) and (33). Of the two boundary conditions needed by the differential equation, we have already discussed one, viz., the trivial condition (18). The second boundary condition must be set at infinity. For this purpose we make use of the velocity constraint $v_{\min } \leqslant c$ discussed in $\S 2.4$ except that we now require $v$ to be exactly equal to $c$ at infinity:

$$
\left(\frac{v_{\min }}{c}\right)_{u \rightarrow \infty}=\left(M \frac{R}{R_{\mathrm{fp}}} \frac{B_{p}}{B}\right)_{u \rightarrow \infty}=1 .
$$

This condition is equivalent to the statement that we pick the physically allowed solution with the minimum level of toroidal field. In the language of Michel (1969), it is the "minimum torque" (or equivalently, minimum energy) solution. Such solutions have the minimum angular momentum and energy flux that connect to asymptotic infinity (Kennel et al. 1983).

The logic for picking this particular solution will become clearer in $\S 3.3 .3$ below. The condition (41) states that the "fast critical surface" or the "light surface," i.e., the point at which the fluid speed is equal to $c$, is located at infinity, and not "beyond infinity" (see Okamoto 1974, who calls this surface the Alfven point). If we are interested in a system that extends to a finite maximum value of $u=u_{\max }$ rather than to infinity, we may wish to use the same condition (41) but applied at $u_{\max }$. This would be equivalent to an outflowing boundary condition on the force-free flow.

When we consider a problem in which field lines are entirely inside the Alfven surface, we need two boundary conditions plus a third condition to determine the eigenvalue $s$. In this case, we have only one boundary condition (eq. [18), and the other two conditions must be set at infinity. Given a particular solution such as those discussed in $\S 3.5$, it is straightforward to come up with the necessary boundary conditions.

\section{ANALYTICAL RESULTS}

\subsection{Blandford's Paraboloidal Solution}

When $\nu=1$, the differential equation (26) has an analytical solution:

$$
\begin{aligned}
T(u) & =\left(1+u^{2}\right)^{1 / 2}-u, \\
P(R, z) & =\left(R^{2}+z^{2}\right)^{1 / 2}-z .
\end{aligned}
$$

This is the self-similar version of the paraboloidal solution of Blandford (1976). The location $u_{c}$ of the Alfven surface is obtained by solving the condition (32), which gives 
$u_{c}=\frac{\left(1-M^{2}\right)}{2 M}$.

The components of the magnetic field are given by

$$
\begin{aligned}
B_{R}(R, z) & =\frac{1}{R}-\frac{z}{R\left(R^{2}+z^{2}\right)^{1 / 2}}, \\
B_{\phi}(R, z) & =-\frac{s M}{R}, \\
B_{z}(R, z) & =\frac{1}{\left(R^{2}+z^{2}\right)^{1 / 2}} .
\end{aligned}
$$

The poloidal projection of a field line with footpoint at $R_{\mathrm{fp}}$ has the shape

$z=\frac{1}{2 R_{\mathrm{fp}}}\left(R^{2}-R_{\mathrm{fp}}^{2}\right)$,

which shows explicitly the parabolic geometry of the field (hence the name of this solution).

Note that the poloidal field structure is independent of the rotation parameter $M$ as well as the eigenvalue $s$. In order to determine $s$, we must apply the boundary condition (41). Substituting equations (44) 46 in equation (36), we find

$$
\left(\frac{v_{\min }}{c}\right)_{u \rightarrow \infty}=\frac{2}{|s|} \text {. }
$$

Thus the boundary condition (41) gives

$s=2$.

Only for this value of $s$ does the fluid velocity $v_{\text {min }}$ asymptotically tend to $c$ at infinity. ( $s=-2$ is also a solution, but this corresponds to a Poynting flux pointed towards the disk and is not of interest.) For $s<2, v_{\text {min }}$ exceeds $c$ beyond a certain value of $u$, and the solutions are unphysical. For $s>2, v_{\min }$ is less than or equal to $c$ as $u \rightarrow \infty$ and it would appear that all the solutions with $s>2$ are physical. Within the framework of the pure self-similar problem, there is nothing to indicate that any one of these solutions is more physical than the others. However, the more complete analysis given by Blandford (1976), which includes a regularity condition as $R \rightarrow 0$, shows that only $s=2$ is consistent with a non-singular solution on the axis. All other values of $s$ that appear to be physically valid within the self-similar analysis are, in fact, unphysical since they require a singular current along the axis. This point will become clearer in $\S$ 3.3.3.

The fact that $B_{R}$ and $B_{z}$ of the paraboloidal solution are independent of $M$ means that the poloidal structure is independent of rotation. Thus, the presence of the azimuthal field $B_{\phi}$, however strong it might be, has no collimating or decollimating effect. Roughly, one could say that the collimating hoop stress associated with the toroidal field is exactly canceled by the decollimating effect of the pressure gradient associated with the same field (see Ostriker 1997).

For the solution with $s=2$, we can calculate the Lorentz factor $\gamma_{\min }$ corresponding to the minimum fluid velocity $v_{\min }$. Assuming $u \gg 1$, we find as a function of distance from the disk plane

$$
\gamma_{\min } \approx \frac{2 M}{\left(1-M^{2}\right)^{1 / 2}}\left(\frac{z}{R_{\mathrm{fp}}}\right)^{1 / 2} \approx \sqrt{2}\left[\frac{R^{2}-R_{\mathrm{fp}}^{2}}{R_{\mathrm{LC}}^{2}-R_{\mathrm{fp}}^{2}}\right]^{1 / 2},
$$

where $R_{\mathrm{fp}}$ is the radius of the foot-point of the field line under consideration. Beskin \& Nokhrina (2006) derive and discuss the asymptotic scaling, $\gamma_{\min } \approx R / R_{\mathrm{A}}$, for a relativistic MHD outflow.

\subsection{Michel's Monopole Solution}

When $\nu=0$, the differential equation 26 again has an analytical solution:

$$
\begin{aligned}
T(u) & =1-\frac{u}{\left(1+u^{2}\right)^{1 / 2}}, \\
P(R, z) & =1-\frac{z}{\left(R^{2}+z^{2}\right)^{1 / 2}} .
\end{aligned}
$$

The components of the magnetic field are given by

$$
\begin{aligned}
B_{R}(R, z) & =\frac{R}{\left(R^{2}+z^{2}\right)^{3 / 2}}, \\
B_{\phi}(R, z) & =-\frac{\Omega}{c} \frac{R}{\left(R^{2}+z^{2}\right)} \\
B_{z}(R, z) & =\frac{z}{\left(R^{2}+z^{2}\right)^{3 / 2}},
\end{aligned}
$$

where, as in the paraboloidal case, we have used the boundary condition (41) to determine the coefficient of $B_{\phi}$. This is the monopole solution of Michel (1973a), with the angular velocity parameter $\Omega$ replacing $M$.

The monopole solution is not really a disk wind solution but rather corresponds to a wind emitted from a rotating star. However, by stitching together copies of the monopole solution with opposite signs in the two hemispheres it is easy to generate a split monopole solution. This has a current sheet in the equatorial plane, just like the other solutions described in this paper, and is more like a disk wind. In both the pure monopole solution and the split monopole solution, the poloidal field is purely radial and its geometry is independent of the angular velocity of the star $\Omega$. Thus, as in the paraboloidal solution, rotation induces field lines to sweep back (see the expression for $B_{\phi}$ ), but it has no tendency to collimate the poloidal field.

The Alfven surface is at a fixed radius $R_{\mathrm{A}}=\Omega / c$ and takes the form of a cylinder (the "light cylinder"). This is not the case for any other value of $\nu$.

\subsection{Asymptotic Power-Law Solution at Large $u$ for General $\nu$}

The two analytic solutions discussed above both have $T(u)$ decaying as a power-law at large $u$; Blandford's paraboloidal solution $(\nu=1)$ goes as $T(u) \propto 1 / u$ and Michel's monopole solution $(\nu=0)$ goes as $T(u) \propto 1 / u^{2}$. We now consider a general value of $\nu$ and seek a power-law solution of the form $T(u) \propto 1 / u^{\mu}$ with positive $\mu$. For convenience, we will refer to such solutions as "paraboloidal" since the field lines have the shape of generalized parabolae (see eq. 55].

In the limit of large $u$, we are well outside the Alfven surface (recall that "outside" means $u>u_{c}$ ), and the terms proportional to $M^{2}$ in equation (26) dominate over the other terms. We may thus neglect the subdominant terms and we may also replace $u^{2}+1$ by $u^{2}$. We are then left with the simpler equation

$u^{2} T T^{\prime \prime}-u^{2} T^{2} / \nu+(3-2 \nu) u T T^{\prime}+\nu(\nu-1)\left(1-s^{2}\right) T^{2}=0,(53$

which has a power-law solution of the form

$T(u) \propto u^{-\mu}, \quad \mu=\nu(s-1), \quad \nu \neq 0,1$. 
The above solution for $\mu$ is not valid when $\nu=0$ or 1 because the term with $s^{2}$ in equation (53) vanishes for these cases. However, we have fully analytic solutions for precisely these values of $\nu$, as discussed in the previous subsections. Here we are interested in other general values of $\nu$. In order to be outside the Alfven surface, we require $T(u)<M^{\nu}$ (as discussed earlier), and this is asymptotically possible only if $\mu$ is positive. Thus we require $s>1$. Using equation (20) we find that the poloidal shape of the field lines is given by

$z \propto R^{s /(s-1)}, \quad R \propto z^{(s-1) / s}$.

The shape is vaguely paraboloidal in the sense that $z$ goes as a power of $R$, though the exponent is not exactly 2 .

Given the above solution for $\mu$, the magnetic field components can be calculated via equations (27) 29) and the fluid velocity $v_{\min }$ can be obtained from equation (36). It is easily verified that any asymptotic power-law solution of the form (54) automatically satisfies the boundary condition $v_{\min }=c$ at $u=\infty$.

Appendix A discusses the properties of these powerlaw solutions in some detail. Equation A14 gives a more accurate estimate of the shape of the field line, including a first order correction term. Expressions are also given for the scalings of the three components of the magnetic field and the minimum Lorentz factor $\gamma_{\min }$. The results for the latter generalize the work of Beskin \& Nokhrina (2006) who consider the perfect paraboloidal case $\nu=1$; however, they analyze the MHD problem whereas we consider the simpler force-free case.

To conclude this subsection, we write down the scalings of various quantities corresponding to two regimes: $\nu>1$ and $\nu<1$. These are the key results of this paper. The scalings are derived in the Appendix and are discussed further in $\S 4$. We also provide additional insight into the boundary condition at infinity.

\subsubsection{Scalings for $\nu \geqslant 1$}

As shown in the Appendix, the case $\nu>1$ is simple. For any given $\nu$, there is only one value of $s$ for which there is a paraboloidal solution; this value is almost exactly equal to $2 / \nu$. The asymptotic form of the solution is

$T(u) \approx u^{-(2-\nu)}$,

and the poloidal shape of a field line is given by

$\frac{z}{R_{\mathrm{fp}}} \approx\left(\frac{R}{R_{\mathrm{fp}}}\right)^{2 /(2-\nu)}$.

We give only the leading order terms here, and the reader is referred to Appendix A.2 for the next order terms. The three components of the magnetic field scale as follows along a field line:

$$
\begin{aligned}
& B_{R}\left(\frac{z}{R_{\mathrm{fp}}}\right) \approx\left(\frac{R_{\mathrm{fp}}}{z}\right)^{(4-\nu) / 2}, \\
& B_{\phi}\left(\frac{z}{R_{\mathrm{fp}}}\right) \approx-M\left(\frac{R_{\mathrm{fp}}}{z}\right)^{(2-\nu) / 2}, \\
& B_{z}\left(\frac{z}{R_{\mathrm{fp}}}\right) \approx\left(\frac{R_{\mathrm{fp}}}{z}\right)^{(2-\nu)} .
\end{aligned}
$$

The minimum Lorentz factor varies with $z$ as $\gamma_{\min } \approx M\left(\frac{z}{R_{\mathrm{fp}}}\right)^{(2-\nu) / 2} \approx M \frac{R}{R_{\mathrm{fp}}}=\frac{R}{R_{\mathrm{A}}}$.

The final expression, $R / R_{\mathrm{A}}$, is identical to the result obtained by Beskin \& Nokhrina (2006).

\subsubsection{Scalings for $\nu<1$}

When $\nu<1$, paraboloidal solutions are present for all values of $s>1$, and there is no longer a unique solution. However, the "correct" solution is still very close to $s=2 / \nu(\S \S 4,5)$, so the scalings given above for $T(u), z / R_{\mathrm{fp}}, B_{R}, B_{\phi}, B_{z}$, are all approximately valid. The minimum Lorentz factor now has a more complicated dependence. We find

$$
\begin{aligned}
\gamma_{\min } & \approx M\left(\frac{z}{R_{\mathrm{fp}}}\right)^{(2-\nu) / 2} \approx \frac{R}{R_{\mathrm{A}}}, \quad \frac{z}{R_{\mathrm{fp}}}<u_{0}, \\
\gamma_{\min } & \approx\left(\frac{z}{R_{\mathrm{fp}}}\right)^{\nu / 2}, \quad \frac{z}{R_{\mathrm{fp}}}>u_{0},
\end{aligned}
$$

where $u_{0} \equiv M^{-1 /(1-\nu)}$. We note that the Beskin \& Nokhrina (2006) scaling $\gamma_{\min } \approx R / R_{\mathrm{A}}$ is valid for small values of $z$, but the scaling is different at large distance. The latter regime may be interpreted as the acceleration produced by field line curvature, whereas the former regime represents acceleration as the result of a "slingshot" effect (V. Beskin, private communication).

\subsubsection{Boundary Condition at Infinity as a Regularity Condition on the Axis}

The paraboloidal solutions described above asymptotically have $\Omega R \gg c$, and their poloidal streamlines are almost precisely cylindrical. Let us, therefore, consider a purely cylindrical force-free problem in which $B_{R}=0$, and $B_{\phi}, B_{z}$ and $\Omega$ are functions only of $R$. The equilibrium condition (GradShafranov equation) for such a flow takes the simple form,

$$
\frac{d B_{z}^{2}}{d R}-\frac{1}{R^{2}} \frac{d}{d R}\left(R^{2} \frac{\Omega^{2} R^{2}}{c^{2}} B_{z}^{2}\right)+\frac{1}{R^{2}} \frac{d}{d R}\left(R^{2} B_{\phi}^{2}\right)=0 .
$$

Asymptotically far from the disk our self-similar solutions have $B_{\phi} \gg B_{z}$, and so the first term is negligibly small; in fact, for the particular self-similar solution with $s=2 / \nu$ discussed in $\S 3.3 .1, B_{z}$ is independent of $R$ at a fixed asymptotic $z$, so this term vanishes exactly. The remaining two terms then give

$B_{\phi}^{2}=\frac{\Omega^{2} R^{2}}{c^{2}} B_{z}^{2}+C$,

where $C$ is an arbitrary integration constant. For a given $B_{z}$, we have an infinite number of solutions, each with a different value of $C$. This is equivalent to the freedom we had in choosing $s$ in $\S \S 3.1,3.2$. However, as we now show, only one of these solutions is well-behaved.

Although we have focused on self-similar solutions in this paper, we imagine that the solutions are modified inside a "core" region at small radii so as to be analytic on the axis. The rotation profile, for instance, might behave as a power law at large $R$ but we expect it to asymptote to a finite constant value as $R \rightarrow 0$. Equation (65) then shows that $B_{\phi} \rightarrow C^{1 / 2}$ on the axis. However, any solution that has a finite $B_{\phi}$ as $R \rightarrow 0$ will have a singular current along the 
axis, which might be considered undesirable. Therefore, let us impose the additional condition that $B_{\phi} \rightarrow 0$ as $R \rightarrow 0$. This can be satisfied only if $C=0$, and so we obtain the unique solution

$\frac{B_{\phi}}{B_{z}}=-\frac{\Omega R}{c}$.

By 65 this relation must be true not only near the axis but at all $R$, including the self-similar region outside the core. Thus, analyticity on the axis picks out a unique solution even in the far-field self-similar zone. Blandford (1976) reached the same conclusion for the specific case $\nu=1$.

In the self-similar region, our solutions have $B_{\phi} \gg B_{z}$, so the quantity on the left-hand side of (66) is equal to $B / B_{p}$, while that on the right is equal to $R / R_{\mathrm{A}}=M R / R_{\mathrm{fp}}$. Thus, the condition (66) is the same as

$M \frac{R}{R_{\mathrm{fp}}} \frac{B_{p}}{B}=1$,

i.e., the same as the condition (41). In other words, the boundary condition $v_{\min } \rightarrow c$ at infinite distance, which we previously argued is a physically motivated boundary condition, is in some sense equivalent to the requirement that the solution be analytic on the axis. This provides additional insight on the boundary condition (41).

\subsection{Power-Law Solution Inside the Alfven Surface}

Consider next the region well inside the Alfven surface. Here we may ignore the terms proportional to $M^{2}$ in equation (26). Let us also assume $u^{2} \gg 1$. The differential equation then simplifies to

$u^{2} T^{\prime \prime}+(3-2 \nu) u T^{\prime}+\nu(\nu-2) T=0$.

This has a power-law solution

$T(u) \propto u^{-\mu}, \quad \mu=-\nu, 2-\nu$.

Since we assume $\nu>0$, the solution $\mu=-\nu$ corresponds to $T(u)$ increasing with $u$. Asymptotically, this gives a solution with a poloidal streamline of the form

$z \propto R^{0}=$ constant.

Thus, as $u \rightarrow \infty$, the field line asymptotes to a constant value of $z$ and the radius $R$ tends to zero. Thus, field lines converge radially onto the $z$-axis. This is clearly unphysical since it implies a non-zero magnetic monopole density on the axis. Interestingly, the self-similar solutions described by Blandford \& Payne (1982) are of this kind and are unphysical.

The second solution in (69), $\mu=2-\nu$, has $T(u)$ decreasing with increasing $u$ (so long as $\nu<2$ ). This asymptotic solution is valid only over the restricted range of $u$ satisfying $u_{c} \gg u \gg 1$. For the specific case of a non-rotating solution, $u_{c} \rightarrow \infty$, and the solution is valid for all $u \gg 1$. The poloidal shape of the field line is given by

$z \propto R^{2 /(2-\nu)}$.

This corresponds to a perfect parabola when $\nu=1$ (Blandford's solution), and is a generalized parabola for other values of $\nu$.

\subsection{Asymptotic Cylindrical Solution}

Consider next solutions in which field lines asymptotically become cylindrical with a finite value of $R$. That is, as $z \rightarrow \infty$, the radius $R$ of each field line tends to a finite value. Equivalently, as $u \rightarrow \infty$, we have both $T^{\prime \prime}$ and $T^{\prime}$ tending to 0 and $T$ tending to a finite value. Note that these conditions are different from the quasi-cylindrical asymptotics we discussed in $\S 3.3 .3$. There, $R / R_{\mathrm{fp}}$ of a field line went to infinity as $u \rightarrow \infty$, whereas here we consider solutions in which $R / R_{\mathrm{fp}}$ remains finite.

Given the above conditions, most of the terms in equation (26) vanish and we immediately obtain

$\left.T(u)\right|_{u \rightarrow \infty}=\left[\frac{(\nu-1)\left(1-s^{2}\right)}{(\nu-2)}\right]^{\nu / 2}$.

The asymptotic radius $R_{\infty}$ of the field line is related to the radius of the footpoint by

$\frac{R_{\infty}}{R_{\mathrm{fp}}}=\left[\frac{(\nu-1)\left(1-s^{2}\right)}{(\nu-2)}\right]^{-1 / 2}$,

and the asymptotic components of the magnetic field are given by

$$
\begin{aligned}
& B_{R}=0, \\
& B_{\phi}=-R^{\nu-2} \nu s M\left[\frac{(\nu-1)\left(1-s^{2}\right)}{(\nu-2)}\right]^{1 / 2}, \\
& B_{z}=R^{\nu-2} \nu\left[\frac{(\nu-1)\left(1-s^{2}\right)}{(\nu-2)}\right] .
\end{aligned}
$$

The azimuthal velocity of the field line is

$$
\left(\frac{v_{\phi}}{c}\right)_{z \rightarrow \infty}=\left[\frac{(\nu-1)\left(1-s^{2}\right)}{(\nu-2)}\right]^{-1 / 2} \text {. }
$$

The above expressions give real numbers only if the quantity in the square parentheses is positive. If $\nu>1$, this requires $s^{2}>1$, and if $\nu<1$, it requires $s^{2}<1$. These are necessary conditions for the existence of cylindrical solutions.

Note that asymptotically cylindrical solutions may be either inside or outside the Alfven surface. Whether a particular solution is inside or outside is determined by whether $R_{\infty}$ is less than or greater than $R_{\mathrm{A}}$, or equivalently, by whether $v_{\phi} / c$ is less than or greater than unity. If the quantity in square parentheses is less than 1, then the solution is outside the Alfven surface. Clearly, this is always the case when $\nu<1$. When $\nu>1$, there is no apparent restriction, though in practice only solutions inside the Alfven surface are present (see $\S 4$ ).

\subsection{Conical Solution}

These are solutions in which $T$ goes to zero at a finite value of $u=u_{f}$, so that field lines asymptotically have a conical shape with $z / R=u_{f}$ as $z, R \rightarrow \infty$. The solution does not exist for $R<z / u_{f}$, i.e., inside the cone. Thus, for force balance, one must supply the required pressure at the surface of the cone. Because this is somewhat unphysical, and also since $v_{\min }>c$ as $u \rightarrow u_{f}$, we do not consider these solutions interesting. Nevertheless, we discuss them briefly for completeness.

As stated above, we have $T \rightarrow 0$ as $u \rightarrow u_{f}$. In this 
limit, the dominant terms in equation (26) are the first and second terms inside the square brackets. Focusing on these, the solution must satisfy

$\nu T T^{\prime \prime}=T^{\prime 2}$,

which has a power-law solution of the form

$T(u) \propto\left(u_{f}-u\right)^{\nu /(\nu-1)}, \quad \nu>1$.

This is the conical solution, which is present only for $\nu>1$.

\section{NUMERICAL SOLUTIONS}

Before describing the numerical solutions, let us recall the properties of Blandford's (1976) paraboloidal solution $(\nu=$ $1, \S 3.1)$.

(i) The poloidal structure of field lines is perfectly parabolic.

(ii) Rotation has neither a collimating nor a decollimating effect on the poloidal configuration of field lines (see Fig. 1).

(iii) For values of $s \geqslant 2$, the solutions are physically acceptable in the limited sense that $v_{\min } \leqslant c$ at all points (solid and dashed lines in Fig. 2). For $s<2$, the solutions have $v_{\text {min }}$ exceeding $c$ beyond a certain value of $u$. (Technically, we should replace $s$ by $|s|$ in these statements, but we are interested only in solutions with positive $s$.)

(iv) Among solutions with $s \geqslant 2$, the particular solution with $s=2$ has $v_{\text {min }}$ asymptotically tending exactly to $c$ (fast critical surface at infinity), and it is also the only solution that can be matched to an analytic core on the axis (§ 3.3.3). In addition, it is the solution with the minimum torque among all solutions that have $v_{\min } \leqslant c$. Thus, this unique solution is the "correct" solution to the self-similar problem. In a sense, the constraint on the fast surface and the analyticity argument provide a physical motivation for Michel's (1969) minimum torque condition.

(v) For the solution with $s=2$, the Lorentz factor increases with distance along the $z$-axis as $\gamma_{\min } \sim$ $M\left(z / R_{\mathrm{fp}}\right)^{1 / 2}$ (eq. 50] Beskin \& Nokhrina 2006).

As we show below (and also in the Appendix), the paraboloidal solution $\nu=1$ acts as a watershed in solution space - solutions on one side $(\nu>1)$ have very different properties compared to those on the other side $(\nu<1)$.

\subsection{Solutions with $\nu>1$}

We have numerically scanned the solution space of equation (26) for the particular choice of parameters $\nu=1.25$ and $M=0.1$. Keeping these parameters fixed, we solved for $T(u)$ corresponding to a range of values of the eigenvalue $s$. Then, using the numerical solution we calculated the components of the magnetic field, the minimum fluid velocity, the minimum Lorentz factor, etc. We identified which solutions are physically acceptable, i.e., have $v_{\min } \leqslant c$ for all $u$ (technically, out to the maximum $u$ to which we integrate, which is $\sim 10^{12}$ ), and which are not, which solutions have power-law, i.e., generalized parabolic, asymptotics (eq. 55) and which have cylindrical asymptotics as $u \rightarrow \infty$, and we also flagged any special solutions that had $v_{\min } \rightarrow c$ as $u \rightarrow \infty$.

As per the discussion in $\S 2.3$, we first checked whether $s^{2} \leqslant 1 /(\nu-1)$. If so, we solved for $u_{c}$, i.e., the position of



Figure 1. Shows the poloidal structure of field lines for the paraboloidal solution, $\nu=1$. The calculations correspond to $M=0.1$ and eight values of $s: 0.5,1.0 \cdots 4.0$. All the solutions have exactly the same poloidal structure (§ 3.1). Contrast this with Figs. 3 and 5.
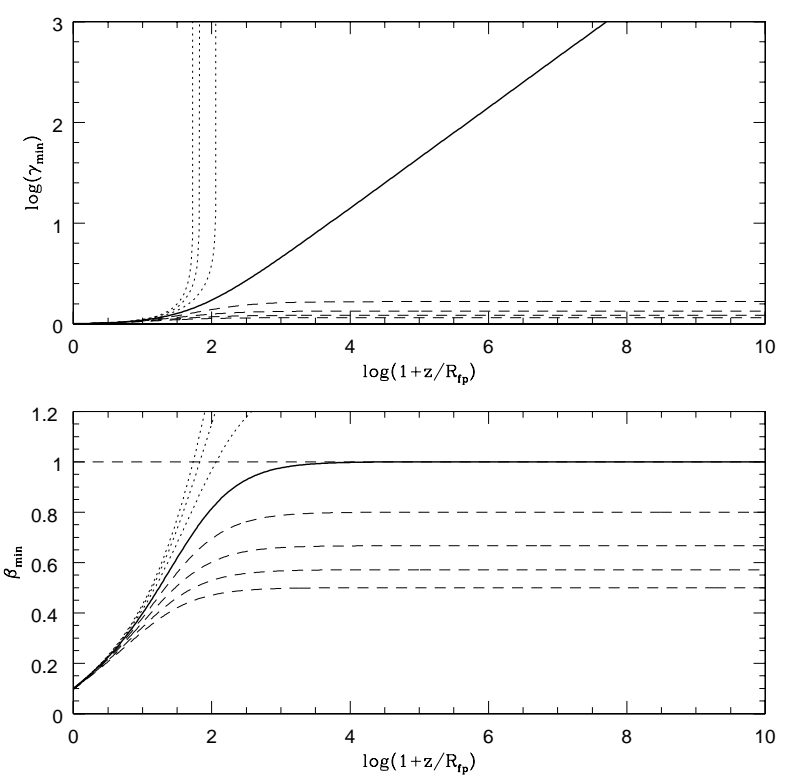

Figure 2. Shows the variation of the minimum Lorentz factor $\gamma_{\min }$ and the minimum velocity $\beta_{\min }=v_{\min } / c$ as a function of distance $z$ from the disk plane for the paraboloidal $(\nu=1)$ solutions of Fig. 1. The thick solid line is the physically interesting solution with $s=2$, whose velocity asymptotically approaches $c$ at infinity. The dotted lines correspond to solutions with $s<2$, which attain minimum velocities exceeding $c$. The dashed lines correspond to solutions with $s>2$, whose velocities remain below $c$ at all $z$. 


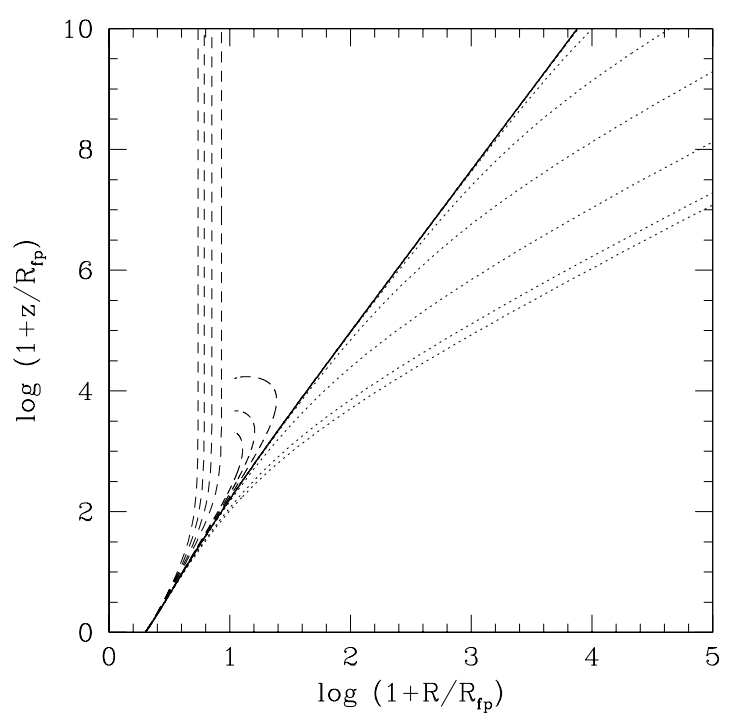

Figure 3. Shows the poloidal structure of field lines for selfsimilar solutions with $\nu=1.25$. The calculations correspond to $M=0.1$ and $s=0.5,1.0,1.5,1.59,1.599,1.5999,1.59996$ (dotted lines), $s=1.5999635948$ (heavy solid line), $s=1.7,1.8,1.9,2.5$, 3.0, 3.5, 4.0 (dashed lines). The heavy solid line is the physically interesting solution that satisfies the boundary condition [41].

the Alfven surface, by integrating equation (26) and applying the conditions (18), (32), (33). In practice, we found it convenient to assume a value of $u_{c}$ and integrate the differential equation (26) from the Alfven surface $u=u_{c}$, where (32) and (33) provide initial conditions, back to $u=0$. At $u=0$ we checked whether the solution satisfied the condition $T(0)=1$. If not, we tried a different value of $u_{c}$ and iterated until the solution converged. We then integrated equation (26) outside the Alfven surface to large values of $u \gg u_{c}$ to complete the solution.

If $s^{2}>1 /(\nu-1)$, we looked for a solution that lives entirely inside the Alfven surface. The only physically acceptable solutions of this kind are those with cylindrical asymptotics. Therefore, we used the discussion in $\S 3.5$ to assign the necessary boundary conditions at infinity. By construction, all of these solutions have $v_{\min }<c$.

Figure 3 shows the poloidal structure of the field lines for various solutions, and Figure 4 shows the corresponding variations of $\beta_{\min }=v_{\min } / c$ and $\gamma_{\min }$ with distance. The thick solid lines correspond to the unique solution that has $v_{\min } \rightarrow c$ as $u \rightarrow \infty$. This solution corresponds to $s \approx 1.6=$ $2 / \nu$ (see Appendix A.1). The poloidal shape of field lines is asymptotically of the form $z \propto R^{2 /(2-\nu)}$ (see eq. [55), i.e., a generalized parabola.

Solutions with $s$ on either side of the above critical value are "unstable" (Appendix A.1; see also Contopoulos 1995a). When $s<2 / \nu$ (shown by dotted lines in Figs. 3 and 4), the solutions have fluid velocities exceeding $c$ and are unphysical. They are all asymptotically conical (see $\S 3.5$ ). Solutions with $s>1 /(\nu-1)^{1 / 2}$ are entirely inside the Alfven surface and have cylindrical asymptotics (dashed lines in Figs. 3, 4). These solutions all have $v_{\min }<c$ as $u \rightarrow \infty$. Over the range
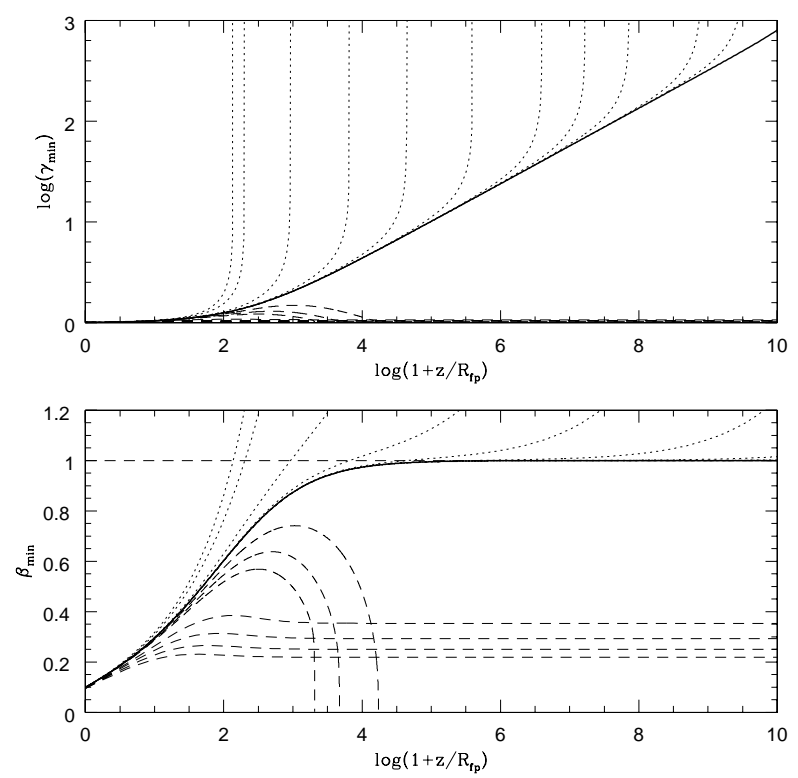

Figure 4. Shows the variation of the minimum Lorentz factor $\gamma_{\min }$ and the minimum velocity $\beta_{\min }=v_{\min } / c$ as a function of distance $z$ from the disk plane for the $\nu=1.25$ solutions of Fig. 3 .

$2 / \nu<s<1 /(\nu-1)^{1 / 2}$, field lines go through the Alfven surface, move out to a maximum radius and then turn back towards the Alfven surface. The solutions become singular when they attempt to recross the Alfven surface.

\subsection{Solutions with $\nu<1$}

Figures 5, 6 show results corresponding to the parameter choice of $\nu=0.75$ and $M=0.1$. As before, we have calculated solutions for a range of values of $s$ and cataloged their properties.

For $s \leqslant 1$ (the first five dotted lines in Figs. 5, 6), there are no asymptotic power-law solutions available (see the discussion in $\S 3.3)$. These solutions are asymptotically cylindrical, superposed with a decaying oscillation, and they are unphysical $\left(v_{\min }>c\right)$. For all values of $s>1$, we have power-law paraboloidal solutions, and these are "stable" in the sense described in Appendix A.1.

Over the range $1<s \lesssim 2.8146$, the solutions have $v_{\min }>c$ somewhere within the range of integration over $u$ and are thus unphysical. The thick solid lines in Figures 5,6 correspond to $s=2.8146$, the lowest value of this parameter for which we can find a physically allowed solution extending over a large range of $u$ (our integrations extended out to a maximum $u \sim 10^{12}$ ). As per equation (55), the poloidal shape of field lines follows $z \propto R^{1.55}$. We note that this critical solution has nearly the same scalings as for the $\nu=1.25$ solution described in the previous subsection, viz., $s \approx 2 / \nu=2.67, z \propto R^{2 /(2-\nu)}=R^{1.6}$, but it is slightly different. The difference arises because of the finite value of $M$ that we have selected. In the limit of small $M$, it turns out that the critical solution satisfies exactly the same scalings as the $\nu>1$ solutions described in the previous subsection (see $\S 4.3$ below). 


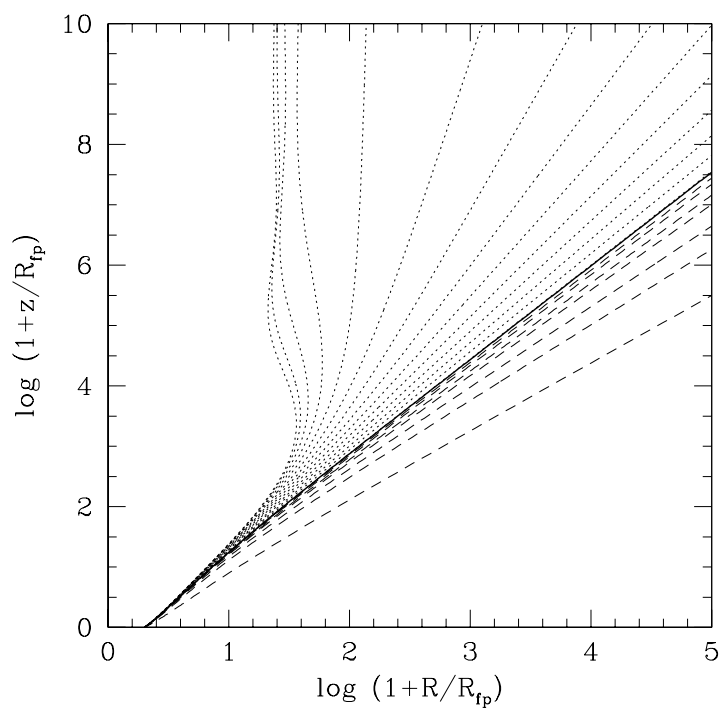

Figure 5. Shows the poloidal structure of field lines for selfsimilar solutions with $\nu=0.75$. The calculations correspond to $M=0.1$ and $s=0.2,0.4 \cdots 2.8$ (dotted lines), $s=2.8146$ (heavy solid line), $s=2.9,3.0,3.2,3.4,4.0,5.0,10.0$ (dashed lines). The heavy solid line is the physically interesting solution. It is the solution with the smallest value of $s$, i.e., the least amount of toroidal field (minimum torque), that satisfies the boundary condition 41].
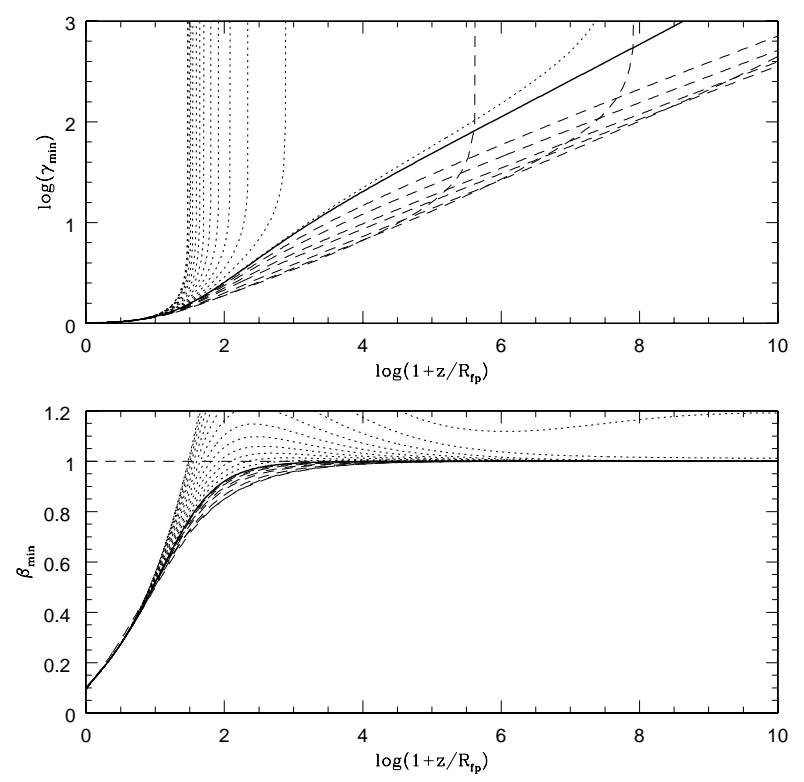

Figure 6. Shows the variation of the minimum Lorentz factor $\gamma_{\min }$ and the minimum velocity $\beta_{\min }=v_{\min } / c$ as a function of distance $z$ from the disk plane for the $\nu=0.75$ solutions of Fig. 5 .

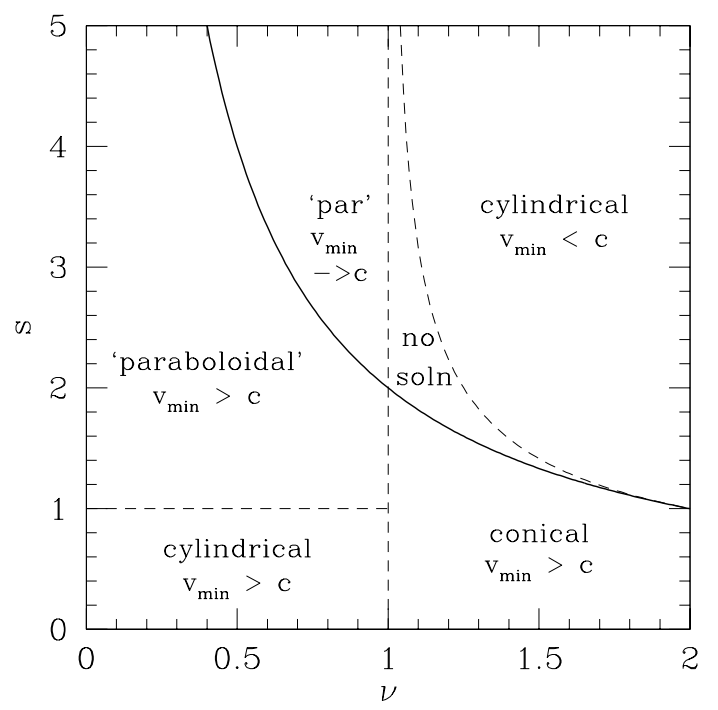

Figure 7. Demarcates the different solution regimes for selfsimilar force-free winds from a thin disk. The regimes are indicated as a function of the similarity index $\nu$ and the field sweepback parameter $s$. The results correspond to the limit $M \ll 1$. The thick line is the locus of solutions with $s=2 / \nu$. These are the physically interesting minimum torque solutions; at each $\nu$, this is the lowest value of $s$ for which a physically valid solution with $v_{\min }<c$ is possible. The various regimes demarcated by dashed lines are described in $\S 4.3$.

For $s>2.8146$ and up to a second critical value $\sim 3.8$ (the first four dashed lines in Figs. 5, 6), all the solutions are physically acceptable, and surprisingly all of them have $v_{\text {min }} \rightarrow c$ as $u \rightarrow \infty$. Thus, there is a continuous family of solutions all of which satisfy the boundary condition (41). However, all these solutions have larger toroidal fields, i.e., larger torques, than the particular solution with $s=2.8146$ described in the previous paragraph, and their Lorentz factors increase more slowly with increasing $z$. Finally, for $s>3.8$ (the last three dashed lines), the solutions revert to being unphysical and the fluid velocity exceeds $c$ over some range of $u$.

We should note that the results of this subsection deviate from those of Contopoulos (1995a) who states that there are no physically viable solutions for $\nu<1$ (see his discussion of solutions of type 1).

\subsection{Survey of Solution Space}

The discussion in the previous two subsections was for two specific values of $\nu$ and for a single choice of $M$. We now briefly put these results in a more general context.

We begin by considering the case in which the rotation parameter $M$ is very small. Figure 7 shows for this case the solution space of self-similar force-free disk winds as a function of the stream function index $\nu$ and the field sweep-back parameter $s$ (compare with Fig. 4 in Contopoulos 1995a, noting that $s \rightarrow-H_{0} c_{n}$ and $\left.\nu \rightarrow x\right)$. The thick solid line corresponds to the relation $s=2 / \nu$. 
For $\nu>1$, paraboloidal solutions are available only on the thick solid line. Below the line, we have unphysical conical solutions. Above the line, within a narrow triangular region, we have no solutions at all. In this region, solutions cross the Alfven surface and then try unsuccessfully to recross the Alfven surface ( $\$ 4.1)$. The solutions are thus unable to reach infinity. Above this forbidden zone, there is an extended region of parameter space where there are asymptotically cylindrical solutions, all of which are physically consistent. The main difference between these solutions and those on the thick solid line is that the cylindrical solutions have $v_{\min }<c$ as $u \rightarrow \infty$.

When $\nu=1$, paraboloidal solutions with physically allowed velocities are available for all points above and on the thick solid line. However, the point $s=2$, which is exactly on the line, is the only solution that is analytic on the axis (§ 3.3.3), and we have argued that it is therefore the correct solution.

For $\nu<1$, the situation is quite interesting. In the limit $M \ll 1$ that we are considering here, physically acceptable paraboloidal solutions are available for all points above and on the thick solid line. Moreover, all these solutions have $v_{\min } \rightarrow c$ for large $u$ and all are analytic on the axis. However, the solutions with $s=2 / \nu$ (the thick solid line in Fig. 7) are still special in that these solutions have the minimum torque among all the physical solutions and also the most rapid acceleration outward. Previously, we associated the condition $v_{\min }=c$ at infinity with analyticity on the axis and also with the minimum torque condition. Now we find a continuum of solutions that have $v_{\min }=c$ at infinity and are analytic, and it is only the requirement of minimum torque that picks out a unique solution. Following Michel (1969), we believe the minimum torque solution is the physically relevant solution. Points below the thick solid line in Fig. 7 are unphysical since $v_{\min }$ exceeds $c$. These unphysical solutions are either paraboloidal or cylindrical, as indicated in Figure 7.

When $M$ is not arbitrarily small, the space of physically allowed solutions is virtually unchanged for $\nu \geqslant 1$. However, for $\nu<1$, the space of allowed solutions shrinks. This is shown by the four dotted lines in the left panel of Figure 8, which correspond (from the left) to $M=$ $0.0001,0.001,0.01,0.1$. In each case, only the region to the right of the dotted line is physically allowed, while the region to the left (even if it is above the thick solid line) has $v_{\min }>c$. Also, for each value of $\nu$, the lowest value of $s$ for which a physically allowed solution is present (the thick segment of the dotted line) is the one with the minimum torque and the most rapid acceleration. In effect, this solution takes on the role of the solution on the thick solid line when $M \ll 1$.

We should note that the above statements about physically allowed and disallowed solutions are based on integrating our solutions out to a large value of $u \sim 10^{12}$ (beyond this, we are not confident of the accuracy of the numerical integration.) However, in practice, one rarely requires a force-free solution to be physical out to such large distances since even very high- $\sigma$ relativistic MHD flows are likely to feel the effects of inertia well before this. It is therefore interesting to look for force-free solutions that are physically reasonable $\left(v_{\min }<c\right)$ out only out to a modest value of $u$. The right panel in Figure 8 shows the allowed region in param-
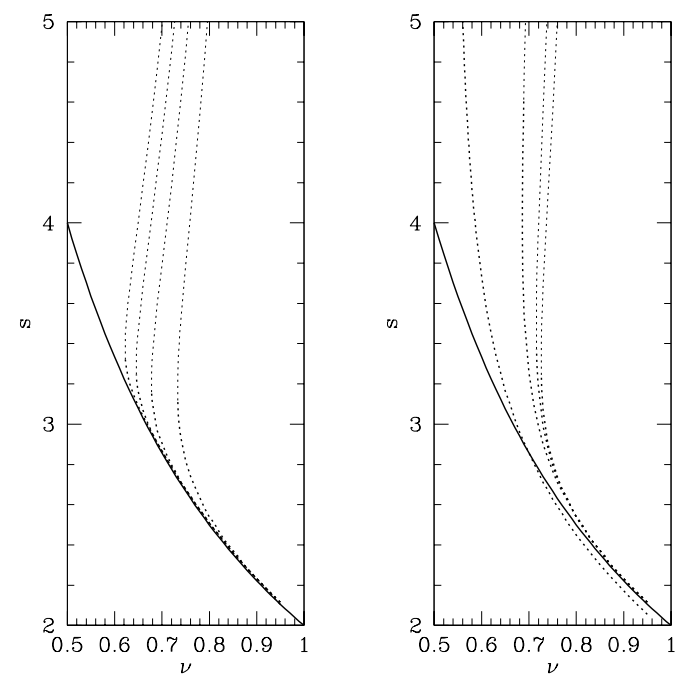

Figure 8. Shows further details of self-similar solutions for $\nu<1$. [Left] The thick solid line is the same as in Fig. 7. In the limit $M \ll 1$, points on and above this line correspond to physically consistent solutions and points below are inconsistent. The four dotted lines show how the region of consistent solutions shrinks for finite values of $M$; from the left, the lines correspond to $M=0.0001,0.001,0.01,0.1$. For each choice of $M$, the region to the right of the corresponding dotted line is the region of consistent solutions. All solutions were numerically integrated from $u=0$ to $u_{\max } \sim 10^{12}$ to determine whether or not they are consistent. [Right] The thick solid line is again the same as in Fig. 7. The dotted lines show the effect of reducing the value of $u_{\max }$ for $M=0.1$; from the left, the lines correspond to $u_{\max }=10^{3}, 10^{5}, 10^{7}, 10^{9}$. For each choice of $u_{\max }$, the region to the right of the corresponding dotted line is the region of consistent solutions.

eter space for different ranges of integration; from the left, $u_{\max }=10^{3}, 10^{5}, 10^{7}, 10^{9}$. Clearly, solutions are allowed over more and more of the parameter space as we reduce the integration range. However, we still have the situation that, for each $\nu$ and integration range, the smallest value of $s$ for which a physically allowed solution is available (the thick segment of the dotted line), i.e., the minimum torque solution, is the one with the largest acceleration.

We now come to a key question. For a given choice of $\nu$ and $M$, i.e., for given self-similar boundary conditions at the equatorial plane, do we know which of the many solutions described here is the correct solution? By correct, we mean the solution that would be picked out by a real system. We have presented a number of arguments to suggest that we should pick the solution with $v_{\min } \rightarrow c$ since this condition appears to be equivalent to analytic behaviour on the axis. But this condition alone is insufficient when $\nu<1$ since there are many solutions satisfying the condition. In the latter case, we have suggested that the correct solution is the one with the minimum torque. The numerical simulations described in $\S 5$ are designed specifically to verify these assertions. 

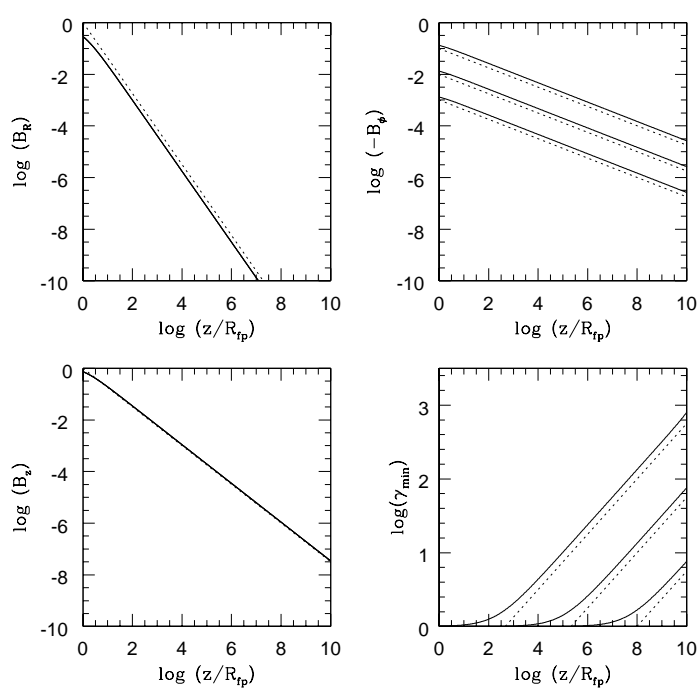

Figure 9. Variations of the three field components, $B_{R}, B_{\phi}$ $B_{z}$, and the minimum Lorentz factor $\gamma_{\min }$ as a function of the coordinate $z$ along a field line. The index $\nu$ is equal to 1.25 , and three solutions are shown, corresponding to $M=0.1,0.01,0.001$, with $s=1.5999635948,1.59999998,1.60000$, respectively. The solid lines show the numerical results and the dotted lines show the approximate scalings given in $\S 3.3 .1$.

\subsection{Numerical Verification of Scalings}

In $\S \S 3.3 .1$ and 3.3.2, we wrote down scaling relations for a number of quantities of interest. We have verified these scalings by comparing the analytical results with numerical solutions.

Figure 9 shows results for $\nu=1.25$ and three values of $M: 0.1,0.01,0.001$. Shown are the three components of the magnetic field, $B_{R}, B_{\phi}, B_{z}$, and the minimum Lorentz factor $\gamma_{\min }$ along a field line. The solid lines correspond to the numerical results and the dotted lines show the analytical scalings. As discussed in Appendix A.2, the analytical results ignore numerical coefficients of order unity and so there are small vertical offsets between the solid and dashed lines. Apart from this, the agreement is good.

Note that the poloidal components of the field, $B_{R}$ and $B_{z}$, are essentially independent of $M$. Thus, rotation has no effect on the poloidal structure, just as in the paraboloidal case discussed in $\S 3.1$. The toroidal component of the field, $B_{\phi}$, does depend on rotation. In fact, its strength is directly proportional to the rotation parameter $M$.

The manner in which the Lorentz factor scales with $z$, viz., $\gamma_{\min } \propto z^{(2-\nu) / 2}$, is independent of $M$, but the actual value of $\gamma_{\min }$ at any given $z$ does depend on $M$. This is because, with decreasing disk rotation, the acceleration of the wind starts at a progressively larger value of $z$. This is not surprising. Acceleration is effective only outside the Alfven surface, because only there does $B_{\phi}$ become the dominant component of the field. When the disk rotates slowly, the Alfven surface is crossed at a larger value of $z$.

Figure 10 shows results for $\nu=0.75$ and the same three values of $M$. For each $M$, we have selected the lowest value
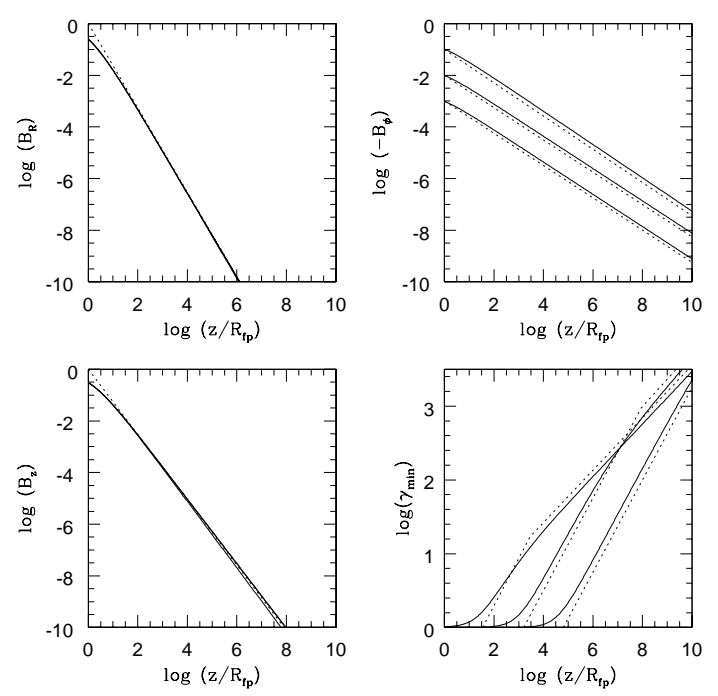

Figure 10. Similar to Fig. 8, but for $\nu=0.75$. The three solutions correspond to $M=0.1,0.01,0.001$, with $s=$ $2.8146,2.67366,2.66710$, respectively. The solid lines show the numerical results and the dotted lines show the approximate scalings given in $\S 3.3 .2$.

of $s$ for which we could obtain a physically valid solution. As discussed earlier, this is the solution with the minimum torque and the largest acceleration. The poloidal structure of the field shows some variation with $M$, but the changes are quite small, so we again find that the poloidal structure is effectively independent of rotation.

The variation of the Lorentz factor with $z$ is rather interesting in this case. We see that there are two regimes: a regime of rapid acceleration soon after a field line crosses the Alfven surface, and a regime of slower acceleration farther out. The scalings given in $\S 3.3 .2$ for $\gamma_{\text {min }}$ agree quite well with the numerical results. Interestingly, the asymptotic Lorentz factor at large $z$ is somewhat larger for a slower spinning disk than for a rapidly spinning disk. This is seen both in the numerical results and in the scaling relations.

\section{TIME-DEPENDENT NUMERICAL SIMULATIONS}

In this section, we discuss the results of time-dependent numerical simulations of force-free nearly self-similar winds. The general relativistic force-free electrodynamics code described in McKinnev (2006a) is used to evolve the axisymmetric force-free equations of motion in a flat spacetime in spherical polar coordinates. This code is an extension of the general relativistic MHD scheme called HARM (Gammie et al. 2003), such that the force-free equations are written as a general set of conservation equations. In the ideal force-free degenerate limit, the force-free equations can be written in a coordinate basis in conservative form as

$\frac{\partial}{\partial t}\left(\sqrt{-g} T_{i}^{t}\right)=-\frac{\partial}{\partial x^{j}}\left(\sqrt{-g} T_{i}^{j}\right)+S(\mathbf{T})$, 
where the index $i$ runs over all three spatial dimensions and the index $j$ runs over the two poloidal spatial dimensions and $\mathbf{T}$ is the stress-energy tensor given by

$T^{\mu \nu}=b^{2} u^{\mu} u^{\nu}+\frac{1}{2} b^{2} g^{\mu \nu}-b^{\mu} b^{\nu}$,

where $b^{\mu}$ is the 4-magnetic field in the frame moving at the drift 4 -velocity given by $u^{\mu}$ and the metric $g^{\mu \nu}$ corresponds to Minkowski space-time with a determinant of $g$. The quantity $S(\mathbf{T})$ is a source term that depends on the coordinate system. These three evolution equations implicitly determine the evolution of the electric field. The magnetic field is governed by the induction equations given by

$\frac{\partial}{\partial t}\left(\sqrt{-g} B^{i}\right)=-\frac{\partial}{\partial x^{j}}\left(\sqrt{-g}\left(b^{j} u^{i}-b^{i} u^{j}\right)\right)$,

where $B^{i}$ is the lab-frame magnetic field that obeys the solenoidal constraint given by

$\frac{1}{\sqrt{-g}}\left(\frac{\partial}{\partial x^{i}}\left(\sqrt{-g} B^{i}\right)\right)=0$

For more details see Komissarov (2002b, 2004); Gammie et al. (2003); McKinnev (2006a).

The force-free version of HARM has been successfully used to study pulsar magnetospheres (McKinnev 2006b), the Blandford-Znajek split-monopole solution (corresponding to $\nu=0$, McKinney 2006a), the Blandford-Znajek paraboloidal solution (corresponding to $\nu=1$ ), and a BlandfordZnajek-type solution with $\nu=3 / 4$ (McKinnev \& Naravan 2006b). The MHD version of HARM has been successfully used to study accretion flows around rotating black holes where nearly force-free jets self-consistently form and are magnetically accelerated to $\gamma \sim 10$ (Gammie et al. 2003, 2004; McKinnev \& Gammie 2004; McKinnev 2006c; McKinnev \& Naravan 2006a).

\subsection{Modelling the Self-Similar Solution}

The divergence of the field strength near the polar axis and at spherical $r=0$ in the self-similar models is problematic for a time-dependent numerical code. Indeed, as we discussed in $\S 3.3 .3$, we assume that the self-similar solution is modified in a core region at small cylindrical radii so as to maintain analyticity around the axis. For analogous reasons, we screen out the divergent region of the numerical solution near spherical $r=0$ in the simulations by introducing an artificial "star." The star is centered at $r=0$ with unit radius and is endowed with a constant angular velocity on its surface equal to the angular velocity in the self-similar disk model at $R=1$. The poloidal field at the surface of the star is chosen to be the same as that given by the self-similar solution on the spherical polar surface $r=1$.

The computational grid is chosen so that at large radii the grid follows the collimating field lines to ensure good resolution. The grid is composed of an arbitrary twodimensional space with coordinate directions $\left\{x_{1}, x_{2}\right\}$. The $x_{1}$ grid lines are mapped to the spherical polar radius $r$ according to

$r=R_{0}+\mathrm{e}^{x_{1}^{n}}$

where $R_{0}=-3, n=10$, and the radius of the grid goes from $R_{\text {in }}=1$ to $R_{\text {out }}=10^{4}$ in arbitrary units of length. The $x_{2}$ grid lines are mapped to the spherical polar $\theta$ according to

$\theta=\left(\frac{\pi}{2}\right)\left(\frac{1+\tan ^{-1}\left[h\left(x_{2}-1 / 2\right)\right]}{\tan ^{-1}[h / 2]}\right)$

where

$h(r)=\left(\frac{r-r_{0}}{r_{1}}\right)^{\alpha}$,

and we choose $r_{0}=0, r_{1}=10$. The index $\alpha$ is chosen to be the same as the index in the scaling of the opening angle of a field line at large radii in the self-similar solution, $\theta_{j} \propto r^{-\alpha}$. From the analytic asymptotic solution given in equation (54), we find that $\alpha=1 / s$.

The resolution for all models is chosen to be $256 \times 128$. The solutions are well-converged compared to low resolution models except very close to the poles where the coordinate singularity of spherical polar coordinates causes minor artifacts that do not affect the results.

\subsection{Obtaining a Force-Free Stationary Solution}

Steady state force-free numerical solutions with no discontinuities or surface currents above the disk surface are found by choosing boundary conditions determined by an analysis of the Grad-Shafranov equation (see, e.g., Bogovalov 1997; Beskin 1997). For solutions that pass through the Alfven surface at some radius, one is required to fix the magnetic field component perpendicular to the conductor $\left(B_{r}\right.$ for the star and $B_{\theta}$ for the disk) and to specify two other constraints at the star or disk, viz., $E_{\phi}=0$, and the value of $\Omega_{F}$, the field line angular velocity.

For axisymmetric, stationary solutions, the frozen-in condition of ideal MHD implies that the field line velocity $v_{i}$ is completely determined by the field $B_{i}$ and field rotation frequency $\Omega_{F}$ (see equation 46 in McKinnev 2006a). Thus, during the simulation the 3-velocity at the stellar surface and on the disk equatorial plane is set to agree with this condition. Such a 3-velocity is generally time-like for points inside the Alfven surface, but outside this region the 3-velocity can sometimes be space-like and unphysical (this is analogous to $v_{\min }>c$ which is discussed extensively in previous sections). In the event that the 3 -velocity becomes space-like during the simulation, the Lorentz factor is locally constrained to a fixed large value as described in section 2.5 of McKinnev (2006a). This safety feature is necessary to handle the violent evolution seen early in the simulations, but it is usually not activated once the solution approaches a stationary state.

In the work described here, the initial conditions in the disk are chosen to correspond to the chosen self-similar solution, described by the parameters $\nu, M$ and $s$. The only difference is that we initially set $B_{\phi}=0$ and allow the code to develop whatever toroidal field it wishes. During the time evolution, $B_{\theta}$ at the disk is held fixed at its initial self-similar value, and the field angular velocity $\Omega_{F}$ is set according to the self-similar model. On the star, the self-similar value of $B_{r}$ is held fixed within $r=1$ and $\Omega_{F}(r=1)$ is set equal to $\Omega_{F}(R=1, z=0)$. Thus the stellar and disk values of $\Omega_{F}$ match at $R=1, z=0$.

The initial non-rotating state is far from steady state, so the model undergoes violent non-stationary evolution. Even- 

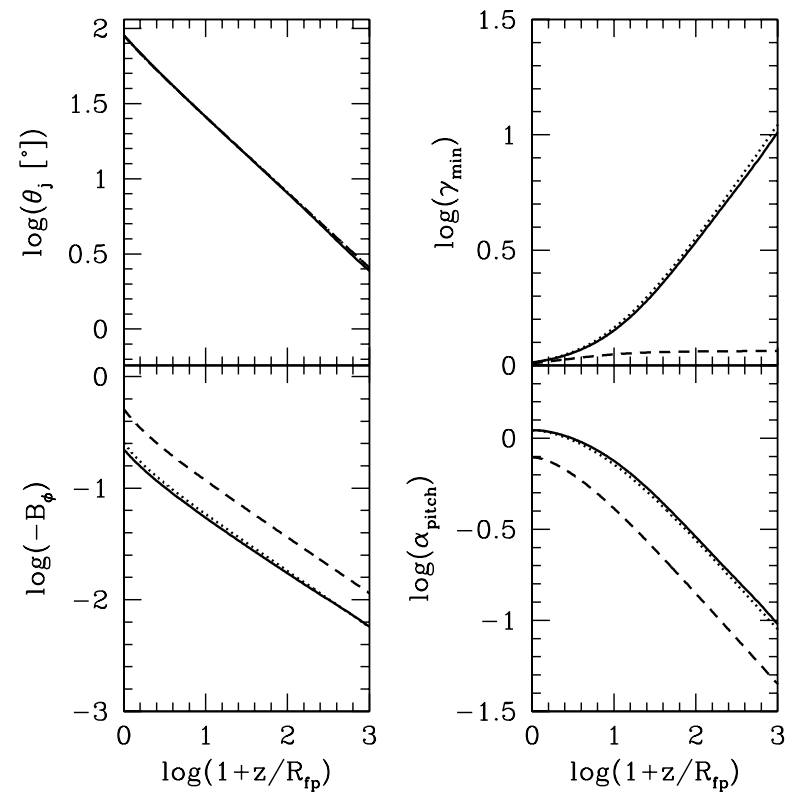

Figure 11. Shows for $\nu=1, M=0.25$, the run of various quantities as a function of $z$ following a particular field line with footpoint at $R_{\mathrm{fp}}=2.0$. The four panels correspond to (i) the opening angle of the field line $\left(\theta_{j}=\tan ^{-1} R / z\right)$, (ii) the minimum Lorentz factor $\left(\gamma_{\min }\right)$, (iii) the toroidal field strength $\left(B_{\phi}\right)$, and (iv) the pitch angle $\left(\alpha_{\text {pitch }}=\tan ^{-1} B_{p} /\left|B_{\phi}\right|\right)$. In each panel, the solid line corresponds to the final converged solution obtained from the time-dependent numerical simulation, the dotted line corresponds to the analytical self-similar solution with $s=2$, and the dashed line corresponds to the analytical solution with $s=4$. It is clear that the numerical solution agrees very well with the $s=2$ analytical solution. This is the smallest value of $s$ for which a physically consistent $\left(v_{\min } \leqslant c\right)$ solution is available, and it is the minimum torque solution.

tually, however, it relaxes to a steady state. All solutions thus found are necessarily stable to Eulerian axisymmetric perturbations.

\subsection{Models with $\nu=1$}

The self-similar model with $\nu=1$ corresponds to the paraboloidal model of Blandford (1976). As we have seen, the poloidal structure of the field is independent of $M$ and $s$. We initialize the simulation with the analytical poloidal solution and set the angular velocity profile in the disk to correspond to the desired value of $M$, viz., $M=0.25$. From the discussion in the previous sections, we know that physically allowed solutions are available for all $s \geqslant 2$, of which the solution with $s=2$ is the one that (i) has $v_{\min }=c$ at large distance, (ii) is analytic on the axis and (iii) has minimum torque. However, recall that $B_{\phi}$ is set equal to 0 initially (corresponding to $s=0$ ) and we allow the code to evolve to whatever value of $s$ it chooses. Which value of $s$ does the time-dependent code select?

Figure 11 shows the results of the numerical model compared against the self-similar analytical model. The plot shows the dependence of various quantities along the particular field line that starts at a foot point radius of $R_{\mathrm{fp}}=2.0$. The top left panel shows the field line's angle away from the

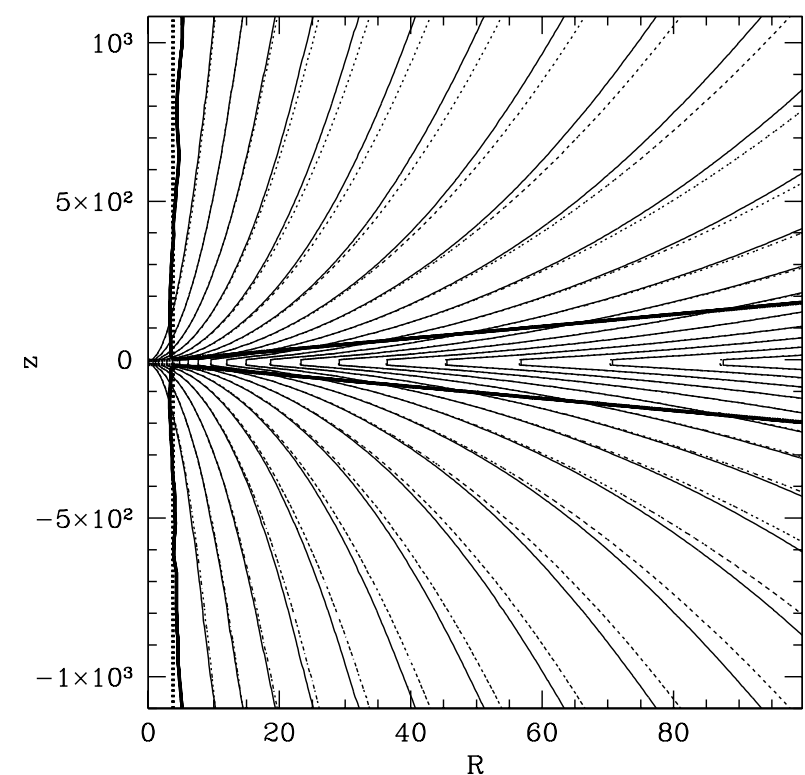

Figure 12. Contours of the stream function $P$ showing the poloidal structure of field lines for $\nu=1, M=0.25$. The thin solid lines show the numerical solution and the dotted lines show the analytical self-similar solution for $s=2$. Note the good agreement. The thick lines are explained in the caption to Fig. 13

polar axis as a function of distance from the disk plane. This quantity is purely a function of the poloidal structure of the field lines, and it agrees very well with the self-similar solution. The other panels show the minimum Lorentz factor, the toroidal field strength, normalized such that $B_{z}(R=1, z=0)=1$, and the pitch angle of the field line defined as

$\alpha_{\text {pitch }} \equiv \tan ^{-1}\left(\frac{B_{p}}{\left|B_{\phi}\right|}\right)$,

where $B_{p}$ is the poloidal field strength. For comparison, the dotted lines show the analytical results for the case $s=2$ and the dashed lines show the corresponding results for $s=4$ (as a counter-example). It is clear that the numerical simulation converges to the model with $s=2$, for which we have

$\alpha_{\text {pitch }} \approx \tan ^{-1}\left(\frac{c}{R \Omega_{F}}\right)$

at large radii.

Figure 12 shows the simulation results for the poloidal field geometry and the location of the Alfven surface. Overlapping the simulation results is the analytical self-similar field geometry and the location of the self-similar Alfven surface. There is excellent agreement for the Alfven surface and reasonable agreement for the disk field lines. Because the simulation has a rigidly rotating star at the center, there is a second branch of the Alfven surface corresponding to the stellar rotation. The position of this branch is in reasonable agreement with the analytical estimate, with minor variations due to under-resolution of the Alfven surface at large radius.

Figure 13 shows the inner region of Figure 12 in order to show how the components of the Alfven surface associated with the star and the disk merge into a single Alfven surface. 


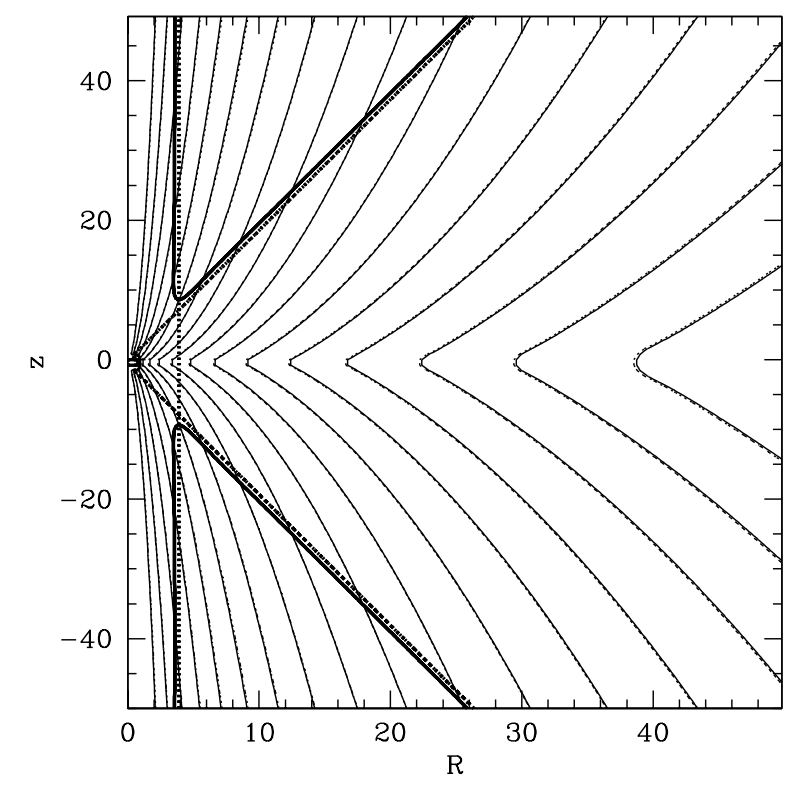

Figure 13. Close-up of the central regions of Fig. 12 highlighting the location of the Alfven surface, shown by the thick solid line. Ignoring the disk, the uniformly rotating star would have its Alfven surface at $R=4$, shown by the thick vertical dotted line. The pure analytical self-similar disk wind solution has its Alfven surface at a fixed angle of $\theta=0.49$ radians away from each polar axis, as shown by the thick sloping dotted lines. It is seen that the Alfven surface in the numerical solution smoothly interpolates between the two analytical Alfven surfaces.

This figure also shows that the field lines are in excellent agreement at small radii.

\subsection{Models with $\nu=0.75$}

Unlike the case of $\nu=1$, self-similar models with $\nu=0.75$ have different poloidal field geometries for each value of $M$ and $s$. The values of $B_{\theta}$ and $\Omega_{F}$ in the disk are specified by the boundary conditions, i.e., the parameters $\nu$ and $M$, and are of course independent of $s$. For this study we choose $M=0.1$ and initialize the simulation with the poloidal solution corresponding to two values of $s: 2.8146$, corresponding to the minimum torque condition, and 5.0, a much larger value. Which value of $s$ will the time-dependent numerical simulation choose?

Figure 14 shows the same information as Figure 11 but for $\nu=0.75$. Regardless of which solution we use to initialize the simulation, the final state selected by the timedependent code corresponds to a solution with $s \approx 2.7$. This is close enough to the special value of $s=2.8146$ that we claim the final solution is the minimum torque solution, in agreement with the proposal of Michel (1969). As discussed in section 4.3 for finite $M$ and for $s$ below a critical value, there is a finite radius beyond which the solution becomes unphysical. Indeed, $s \approx 2.7$ leads to $v_{\min }=c$ at a finite radius somewhat smaller than the size of the simulation box. The simulation does reach $v_{\text {min }}=c$ for $r \sim 10^{3}$, although the numerical method would have to be improved to verify that this is not just numerical error.
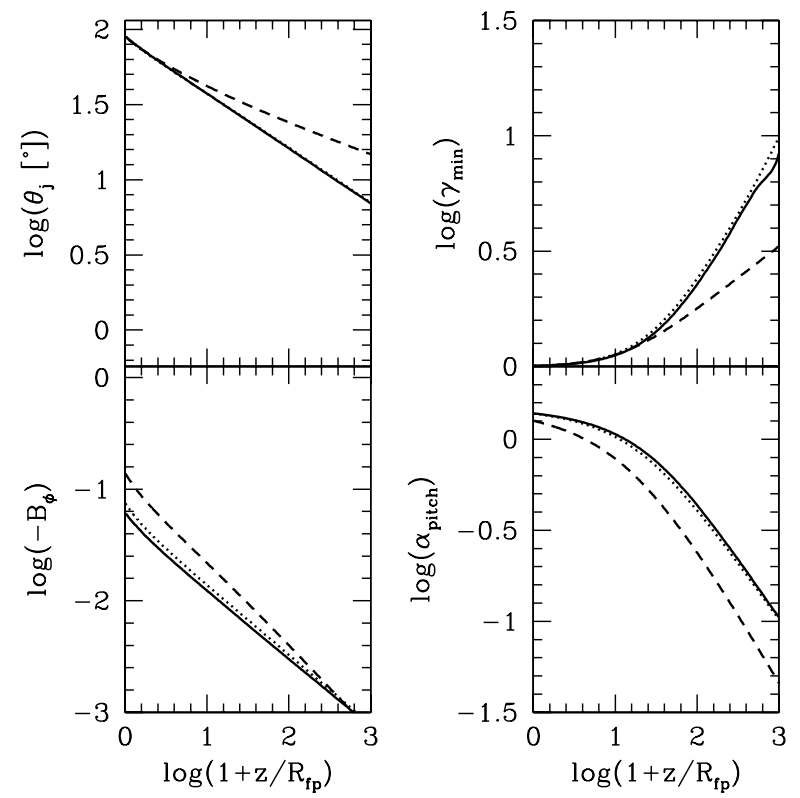

Figure 14. Similar to Fig. 11$]$ but for $\nu=0.75$. The numerical model (solid lines) agrees well with the analytical self-similar solution with $s=2.7$ (dotted lines), but not with the self-similar solution with $s=5.0$ (dashed lines). The former value of $s$ is approximately the smallest for which a physically consistent solution $\left(v_{\min } \leqslant c\right)$ is possible, i.e., it is the minimum torque solution.

\subsection{Models with $\nu=1.25$}

Like self-similar models with $\nu=0.75$, models with $\nu=1.25$ have different poloidal field geometries for each value of $M$ and $s$, while the values of $B_{\theta}$ and $\Omega_{F}$ in the disk are the same for each $s$. For this study we choose $M=0.4$ and initialize the simulation with the self-similar poloidal solution corresponding to two values of $s$ : 1.6 , which corresponds to the paraboloidal solution on the thick solid line in Figure 7, and $s=2.5$, which corresponds to an asymptotically cylindrical solution. Again, the interesting question is which solution will be picked by the time-dependent numerical simulation.

Figure 15 shows the same information as Figure14 but for $\nu=1.25$. Clearly the time-dependent code has chosen a solution close to the paraboloidal solution with $s \approx 1.6$. Even when we start the simulation with the cylindrical solution, the field lines spontaneously open out and become paraboloidal with $s=1.6$. The final solution is the minimum torque solution for this problem.

Note that the behaviour at large radius of the timedependent solution that starts with $s=2.5$ is difficult to follow because the solution undergoes quite a large change from cylindrical to paraboloidal streamlines. Thus one underresolves either the initial solution or the evolved final solution at large radii. For such models we performed two simulations with a grid that resolves one or the other type of field geometry, and found that both resulted in the same final solution with $s \approx 1.6$. 

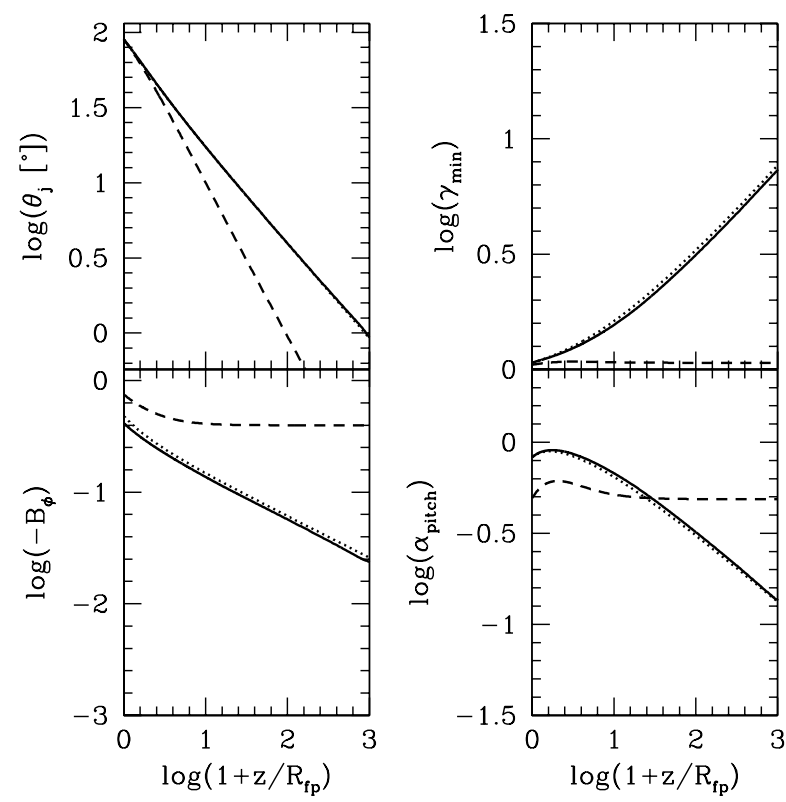

Figure 15. Similar to Fig. 11 but for $\nu=1.25$. The numerical model (solid lines) agrees well with the analytical self-similar solution with $s=1.6$ (dotted lines), but not with the self-similar solution with $s=2.5$ (dashed lines). The former value of $s$ is the smallest for which a physically consistent solution $\left(v_{\min } \leqslant c\right)$ is possible, i.e., it is the minimum torque solution.

\subsection{Summary of Time-Dependent Simulation Results}

In summary, the time-dependent force-free numerical simulations reveal the following:

(i) For a given choice of $\nu$ and $M$, a self-similar force-free system spontaneously seeks the smallest value of the eigenvalue $s$ for which a physically consistent solution $\left(v_{\min } \leqslant c\right)$ is possible throughout the finite numerical grid. Since $s$ measures the strength of the toroidal field, an equivalent statement is that the system chooses the configuration that has the least sweepback of field lines in the toroidal direction, or the minimum torque.

(ii) The final converged numerical solution matches very well the analytical self-similar solution that corresponds to this value of $s$. There is close agreement in the profiles of the components of the magnetic field, $B_{R}, B_{\phi}, B_{z}$, and the minimum Lorentz factor $\gamma_{\min }$.

\section{SUMMARY AND DISCUSSION}

With a view ultimately to understanding the nature of MHD outflows from relativistic accretion disks around spinning black holes, we have considered in this paper the much simpler problem of a self-similar force-free outflow from an infinitely thin rotating equatorial disk. The problem is mathematically described in terms of two dimensionless numbers: (i) $\nu$, the radial index of the stream function $P$ on the equatorial plane (eq. 14), which is defined such that the magnetic field scales as $R^{\nu-2}$ on this plane; (ii) $M$, a rotation parameter, defined such that the azimuthal rotation velocity on the equatorial plane is $M c$, independent of $R$ (eq. 21).
Given these two parameters, the self-similar problem naturally leads to two dimensionless eigenvalues: (i) $s$, which describes the degree of sweepback of the magnetic field lines at the equatorial plane (eq. 23); (ii) $u_{c}$, which gives the value of $z / R$ at the Alfven surface (also called the light cylinder in the pulsar magnetosphere problem). The eigenvalues are obtained by solving the differential equation [26) with appropriate boundary conditions.

Although this is an extremely simple model, the solution space is nevertheless surprisingly rich. We find generalized paraboloidal solutions ( $\S 3.3$ ), cylindrical solutions (§ 3.5), conical solutions ( $\S 3.6)$, and even converging solutions $(\S 3.4)$. Moreover, some of the solutions have field lines crossing the Alfven surface, while others live entirely inside the Alfven surface. Numerical examples of the different kinds of solutions are shown in Figures 1-6, and the regions of parameter space over which the different kinds are found are indicated in Figures 7 and 8. In $\S \S 3.3 .1,3.3 .2$ we discuss scaling relations for paraboloidal solutions, and in Figures 9 and 10 we show that these analytical scalings agree well with numerical solutions.

Given the bewildering variety of solutions, we are faced with the question: which is the "correct" solution for a given choice of $\nu$ and $M$ ? In an attempt to make some headway on this question, we may wish to impose the condition that the minimum fluid velocity $v_{\min }$, as defined in $\S 2.4$, should not exceed $c$ anywhere within the flow. However, this condition only eliminates half the parameter space of solutions (see Figs. 7, 8). Requiring in addition that the solution should be analytic on the axis, we find this corresponds to the desirable condition $v_{\min } \rightarrow c$ asymptotically far from the disk (§ 3.3.3). This leads us to the stronger condition $v_{\min }=c$ at infinity instead of just $\leqslant c$, and it gives us a unique solution for all $\nu \geqslant 1$. However, for $\nu<1$, it still leaves us with a continuous family of physically valid solutions which spans, for each choice of $\nu$ and $M$, a range of values of $s$. To break this residual degeneracy, we are compelled to invoke yet another condition, viz., that the correct solution is the one with the minimum toroidal field, i.e., the minimum torque (Michel 1969). This latter condition subsumes the $v_{\min } \rightarrow c$ condition, and gives a unique solution for all values of $\nu$. It is, however, less well motivated than the analyticity condition on the axis or the requirement that the fast surface be located at infinity. We are thus led to use axisymmetric numerical simulations to verify which solution is picked out by the time-dependent system.

$\S 5$ describes these numerical simulations and summarizes the results (see Figs. 11-15). For each of the three representative combinations of $\{\nu, M\}$ that we analyze, we find that the numerical models converge to a unique final steady state, which is independent of the initial starting state. In all cases, the final state has a value of $s$ equal to the smallest value of this parameter for which our analytical model gives a physically consistent solution (with $v_{\min } \leqslant c$ over the domain of interest), i.e., the solution with the minimum torque. This is also the solution with the largest acceleration.

One interesting question is prompted by the numerical simulations. In the mathematical analysis described in this paper and also in the physical discussion of the previous paragraphs, we assigned great importance to the point at infinity, or at the edge of the grid, and assumed that one or more important boundary condition is set there. 
This is a natural consequence of working with the steady state problem which involves analyzing the elliptic GradShafranov equation. The numerical simulations, however, work with the hyperbolic time-dependent force-free equations. Surprisingly, in the numerical simulations, the flow chooses the more-or-less correct configuration for the magnetic field near the equatorial plane long before any signals (moving at the speed of light) have reached the outer boundary of the grid, or even reached the Alfven surface. Thus, the time-dependent problem appears not to require the point at infinity to set up the local flow. Exactly how does it do this? One answer is that the condition $v_{\min } \rightarrow c$ at infinity is equivalent to analyticity on the axis, i.e., the absence of a singular current on the axis (§3.3.3). The numerical code forbids a singular current on the axis (at all $z$ ) and would therefore naturally pick out such solutions. For $\nu<1$, we require an additional condition that the solution has the minimum torque (or maximum acceleration). This again is a condition that could, in principle, be applied at the equatorial plane and perhaps this is how the code is able to pick the correct solution promptly.

Ultimately, we suspect that only a perturbation analysis of the self-similar steady solutions we have obtained in this paper will provide real understanding of which solution is correct for each situation. It could be that the minimumtorque/maximum-acceleration solution is the only one that is stable to axisymmetric perturbations.

The quasi-analytic work we presented is closely related to that of Contopoulos (1995a), who investigated self-similar force-free flows in order to better understand MHD solutions obtained by Li et al. (1992) and Contopoulos (1994). Our work differs from Contopoulos (1995a) in at least two ways: (i) We have identified a larger set of solutions ; (ii) Contopoulos (1995a) suggests that the value of $-s M$ ( $H_{0}$ in his notation) has to be fine-tuned in order to obtain solutions that reach asymptotic infinity with high Lorentz factors. For a physical, time-dependent solution, he suggests that this fine-tuning corresponds to the requirement that waves propagate from asymptotic infinity and reach the origin, and this would take an infinite amount of time. He suggests, therefore, that such solutions are inapplicable as physical models.

On the other hand, following Blandford (1976) for $\nu=$ 1 , we have been able to identify a regularity condition on the polar axis that leads to a unique solution for each $\nu \geqslant 1$, and we use the minimum-torque (minimum-energy) condition to choose a unique solution for each $\nu<1$. As discussed above, we confirmed these conditions as natural by performing time-dependent numerical simulations that generate solutions that are, by construction, regular on the polar axis and necessarily stable. These simulations indicate that the minimum-torque condition is the natural condition and that there is no fine-tuning required to obtain solutions that reach asymptotic infinity with $v_{\min } \rightarrow c$. However, for $M$ close to unity the solutions with $\nu<1$ are indeed sensitive to the value of $s$ (Fig. 8). As applied to astrophysical winds, this may indicate that for disks with $\nu<1$ the force-free approximation is difficult to maintain at arbitrarily large distances.

Another important issue is how the self-similar forcefree solutions described in this paper are related to selfsimilar MHD solutions such as those discussed by Li et al. (1992) and Vlahakis \& Konigl (2003). We anticipate that MHD outflows that start out with high values of the magne- tization parameter $\sigma$ will closely follow one of our force-free solutions out to a certain distance before plasma inertia induces deviations. Exactly which of our force-free solutions is picked by the MHD flow? Following the work of Goldreich \& Julian (1970), one suspects that MHD would automatically single out the minimum torque force-free solution, but this remains to be seen. It is also interesting to ask exactly where the MHD solution would start deviating from the force-free solution.

Returning to the results obtained in this paper, we note that, to a good approximation, the thick solid line in Figure 7 gives the minimum torque solution for any $\nu$ and $M$. The eigenvalue $s$ is given approximately by $s \approx 2 / \nu$, and the magnetic field components and the Lorentz factor have the approximate asymptotic scalings given in $\S \S 3.3 .1,3.3 .2$. Some of these results have been derived by other authors (e.g., Beskin \& Nokhrina 2006), but some appear to be new. The unification of $\nu \geqslant 1$ models and $\nu<1$ models is another relevant contribution of the present paper. Our recent work (McKinney \& Narayan 1995a,b) has shown that GRMHD models have currents and fields described by $\nu \approx 3 / 4$ for a wide range of conditions. It is therefore important to understand the properties of self-similar models with this particular value of the index, originally introduced by Blandford \& Payne (1982).

An interesting result of our analysis is that rotation has almost no effect on the poloidal structure of field lines. This was known to be the case for the split monopole solution of Michel (1973a) and the $\nu=1$ solution of Blandford (1976), but we now find that it is true for the entire family of selfsimilar solutions considered in this paper. It suggests that the collimation of astrophysical jets is not the result of the toroidal field associated with rotation. In our solutions, collimation seems to be produced by the poloidal field itself. In effect, each field line is collimated by the pressure associated with field lines further out. However, this result is for the specific self-similar model we have considered, which has a flat rotation curve. It remains to be seen if the results carry over to a disk with a Keplerian rotation profile.

In contrast to the case of collimation, our models show that rotation and toroidal field are critical for accelerating the force-free wind. Serious acceleration begins only when the toroidal component of the magnetic field dominates over the poloidal component, which happens only after a field line crosses the Alfven surface. The larger the rotation of the disk, the closer the Alfven surface is to the foot-point of a field line, and the sooner strong acceleration is initiated. This is illustrated in Figures 9 and 10.

Finally, we note that the force-free winds we have considered in this paper are highly idealized, and their relevance to real disk winds is unclear since MHD turbulence within the disk may tangle up such large-scale fields (McKinnev 2005; McKinnev \& Naravan 2006b). Our hope is that some of the analytical results and qualitative insights obtained here may carry over to more realistic MHD models of winds.

\section{ACKNOWLEDGMENTS}

We are grateful to Vasily Beskin and the referee, Serguei Komissarov, for a detailed reading of the paper and for numerous helpful comments. This work was supported in part 
by NASA grant NNG04GL38G. JCM was supported by an Institute for Theory and Computation Fellowship and AJF by a Harvard Junior Fellowship.

\section{APPENDIX A: ANALYSIS OF PARABOLOIDAL SOLUTIONS}

In this Appendix we focus on paraboloidal solutions and derive a few asymptotic results in the limit of large $u=$ $z / R$. We begin by separating out equation (26) into terms of different orders:

$$
\begin{aligned}
u^{2} T T^{\prime \prime} & -u^{2} T^{2} / \nu+(3-2 \nu) u T T^{\prime}+\nu(\nu-1)\left(1-s^{2}\right) T^{2} \\
& -M^{-2} T^{(\nu+2) / \nu}\left[u^{2} T^{\prime \prime}+(3-2 \nu) u T^{\prime}+\nu(\nu-2) T\right] \\
& +T T^{\prime \prime}-T^{2} / \nu \\
& -M^{-2} T^{(\nu+2) / \nu} T^{\prime \prime}=0 .
\end{aligned}
$$

By "paraboloidal" solutions, we mean those in which $T(u)$ goes as $u^{-\mu}$ with a positive value of $\mu$ as $u \rightarrow \infty$. We will concentrate on the region well outside the Alfven surface, where $T(u) \ll M^{\nu}$. It is obvious that the dominant terms in equation (A1) in this region are those listed in the first line, and so we begin with an analysis of these terms. The single term in the fourth line is always much smaller than the others and we will neglect it. The terms in lines 2 and 3 are of intermediate importance and we consider them as needed.

The first line of equation A1 is homogeneous and is identical to equation (53) in the main text. It has two independent power-law solutions. We require a decaying solution, and such a solution exists only when $s>1$ (as in the main text, we restrict our attention to positive values of $M$ and $s$ ). The solution is given by

$T(u) \sim u^{-\mu}, \quad \mu=\nu(s-1)$.

In the following two subsections, we consider the effect of small perturbations on this solution and calculate the next order term in a power series expansion.

\section{A1 Perturbation Analysis of the Homogeneous Equation}

Focusing still on just the homogeneous equation represented by the first line of (A1), consider small perturbations around the power-law solution A2:

$T(u) \approx u^{-\mu}+\epsilon(u), \quad \mu=\nu(s-1), \quad u \gg u_{c}$.

We will assume that $\epsilon(u) \ll u^{-\mu}$. Substituting A3 in A1 and retaining only the leading terms, we obtain

$$
\begin{aligned}
0 & =u^{2} \epsilon^{\prime \prime}+\left(\frac{2 \mu}{\nu}+3-2 \nu\right) u \epsilon^{\prime} \\
& +\left[\mu(\mu+1)-(3-2 \nu) \mu+2 \nu(\nu-1)\left(1-s^{2}\right)\right] \epsilon
\end{aligned}
$$

This equation has power-law solutions of the form $\epsilon \sim u^{-\xi}$, with two values of $\xi$ :

$\xi_{1}=\mu, \quad \xi_{2}=\mu\left(\frac{2}{\nu}-1\right)+2(1-\nu)$.

The first solution is trivial - it merely reproduces the leading order term in equation a and carries no new information. The second solution is, however, interesting. It gives an asymptotic behaviour for $T(u)$ of the form $u^{-\mu}\left(1+C u^{-\left(\xi_{2}-\mu\right)}\right)$, where $C$ is a constant and

$\xi_{2}-\mu=2(1-\nu)\left(\frac{\mu}{\nu}+1\right)$.

We now consider the stability of the solution; we mean stability in a mathematical sense, not dynamical. The perturbation $\epsilon(u)$ will remain smaller than the primary term $u^{-\mu}$ with increasing $u$ only if $\xi_{2}-\mu>0$, i.e., only if $\nu<1$. Thus, so long as $\nu<1$, the paraboloidal solution is "stable" for any positive value of $\mu$, i.e., for any $s>1$. This is confirmed by Figure 5, where we see that all the solutions with $s>1$ are well-behaved and have power-law, i.e., paraboloidal, behaviour as $u \rightarrow \infty$.

When $\nu>1$, however, we see that $\xi_{2}-\mu$ is always negative, and so perturbations grow with increasing $u$ and ultimately dominate over the leading order term $u^{-\mu}$. This is a sign that the solution is "unstable," as confirmed by Figure 3. Apart from the cylindrical solutions, we see that nearly all the other solutions are either conical, i.e., $T(u)$ goes to 0 at a finite $u=u_{f}$, or singular, i.e., $T(u)$ attempts, and fails, to recross the Alfven surface. Obviously, none of these solutions is physically satisfactory. There is, however, one (and only one) paraboloidal solution which manages to avoid the instability; it is shown as the thick solid line in Figure 3. To understand how this special solution manages to avoid growing perturbations, we note that perturbations to the primary solution $u^{-\mu}$ are generated by the terms in lines 2-4 of equation A1 So let us briefly take a look at these terms.

Consider the terms in the second line of equation (A1). They add up to zero when $T(u)$ has the power-law form $u^{-(2-\nu)}$. Thus, for this particular power-law, these terms do not introduce any perturbations at all to the solution. In general, the primary power-law solution $u^{-\mu}$ is not of this special form. However, there is one particular case when the same power-law solution satisfies both lines 1 and 2 of equation (A1). This is when

$s=\frac{2}{\nu}, \quad \mu=2-\nu$.

So for this one value of $s$, line 2 does not introduce any perturbations to the primary $u^{-\mu}$ dependence of $T(u)$. We would therefore expect the solution for this particular value of $s$ to be stable. It is gratifying to see that the special solution shown by the thick solid line in Figure 3 does indeed correspond to $s$ being very nearly equal to this special value, and the solution does behave asymptotically as $u^{-(2-\nu)}$. Of course, we only considered the first two lines of A1. For the above special solution, line 3 is of a lower order than line 2 , but it is not completely negligible. Therefore, the paraboloidal solution does not correspond exactly to the value of $s$ given in (A7), but it is very close.

In summary, for $\nu<1$, a paraboloidal solution is available for all $s>1$, while for $\nu>1$ it is available for only one specific value of $s$ which is nearly equal to $2 / \nu$.

\section{A2 Power Series Expansion and Lorentz Factor}

In this subsection, we ignore line 2 of equation (A1). When $\nu>1$, we showed above that the contribution of this line vanishes for the only paraboloidal solution available. When 
$\nu<1$, this line is sub-dominant relative to line 3 for most paraboloidal solutions of interest to us, especially the ones on and above the thick solid line in Figure 7.

Consider, therefore, the differential equation consisting of lines 1 and 3 of equation (A1). As before, consider a solution of the form A3, where we assume that the second term is much smaller than the first. When this solution is substituted in line 1, the zeroth order terms cancel and the first order terms take the form given in the left hand side of equation A4. On the other hand, the zeroth-order terms give a non-vanishing contribution when substituted in line 3. Thus, collecting terms, we obtain the following differential equation for $\epsilon$ :

$$
\begin{aligned}
& u^{2} \epsilon^{\prime \prime}+\left(\frac{2 \mu}{\nu}+3-2 \nu\right) u \epsilon^{\prime} \\
+ & {\left[\mu(\mu+1)-(3-2 \nu) \mu+2 \nu(\nu-1)\left(1-s^{2}\right)\right] \epsilon } \\
= & -\left[\mu(\mu+1)-\frac{\mu^{2}}{\nu}\right] u^{-\mu-2}, \quad \mu=\nu(s-1) .
\end{aligned}
$$

Solving this, we obtain

$\epsilon(u)=-C u^{-(\mu+2)}$,

where $C$ is a coefficient which is expressed in terms of $\nu, \mu$ and $s$. In the rest of this subsection we will ignore coefficients of order unity, such as $C$, since our primary interest is in the scalings of various quantities. Thus, we write the solution for $T(u)$ as

$T(u) \approx u^{-\mu}\left(1-\frac{1}{u^{2}}\right)$

Clearly, this solution gives the first two non-zero terms of a power series expansion in $1 / u$ of the paraboloidal solution (note that the term proportional to $1 / u$ vanishes), i.e., the first two nonvanishing terms of an expansion around the point at infinity. For many purposes, the first term is good enough, but the second term is essential for estimating the Lorentz factor of the force-free outflow, as we now discuss. Incidentally, to get an idea of the error we make by neglecting coefficients of order unity, note that the power series expansions of the two analytic solutions given in $\S \S 3.1$ and 3.2 are given by

$\begin{array}{ll}T(u) \approx \frac{1}{2 u}\left(1-\frac{1}{4 u^{2}}\right), & \nu=1, \quad \mu=1, \\ T(u) \approx \frac{1}{2 u^{2}}\left(1-\frac{3}{4 u^{2}}\right), & \nu=0, \quad \mu=2 .\end{array}$

The factors $1 / 2,1 / 4$ and $3 / 4$ are omitted when we write the series expansion approximately as in A10,

The stream function $P(R, z)$ corresponding to the above solution is

$P(R, z)=R^{\nu} T(u) \approx \frac{R^{\mu+\nu}}{z^{\mu}}\left(1-\frac{R^{2}}{z^{2}}\right)$.

Since $P$ is constant on field lines, we can solve for the shape of a field line with footpoint at $R_{\mathrm{fp}}$ :

$$
\begin{aligned}
\frac{R}{R_{\mathrm{fp}}} & \approx\left(\frac{z}{R_{\mathrm{fp}}}\right)^{\mu /(\mu+\nu)}\left[1+\left(\frac{R_{\mathrm{fp}}}{z}\right)^{2 \nu /(\mu+\nu)}\right] \\
& =\left(\frac{z}{R_{\mathrm{fp}}}\right)^{(s-1) / s}\left[1+\left(\frac{R_{\mathrm{fp}}}{z}\right)^{2 / s}\right],
\end{aligned}
$$

where, in the final expression, we have used equation A2 to substitute for $\mu$. From equations (27), (28), 29), we can estimate the three components of the magnetic field:

$$
\begin{aligned}
B_{R}(R, z) & \approx \frac{R^{\mu+\nu-1}}{z^{\mu+1}} \\
B_{\phi}(R, z) & \approx-M \frac{R^{\left(\nu^{2}-2 \nu+\mu \nu-\mu\right) / \nu}}{z^{\mu(\nu-1) / \nu}}\left(1-\frac{R^{2}}{z^{2}}\right), \\
B_{z}(R, z) & \approx \frac{R^{\mu+\nu-2}}{z^{\mu}}\left(1-\frac{R^{2}}{z^{2}}\right) .
\end{aligned}
$$

We have written the first two terms for $B_{\phi}$ and $B_{z}$, but only the leading term for $B_{R}$ since this component of the field is very weak at large $u$.

Consider now the variation of the field components as a function of $z$ along a given field line. To calculate this, we substitute for $R$ from equation (A14):

$$
\begin{aligned}
& B_{R}\left(\frac{z}{R_{\mathrm{fp}}}\right) \approx\left(\frac{R_{\mathrm{fp}}}{z}\right)^{(2 s-1) / s}, \\
& B_{\phi}\left(\frac{z}{R_{\mathrm{fp}}}\right) \approx-M\left(\frac{R_{\mathrm{fp}}}{z}\right)^{(s-1) / s}\left[1-\left(\frac{R_{\mathrm{fp}}}{z}\right)^{2 / s}\right](, \mathrm{A} \\
& B_{z}\left(\frac{z}{R_{\mathrm{fp}}}\right) \approx\left(\frac{R_{\mathrm{fp}}}{z}\right)^{2(s-1) / s}\left[1-\left(\frac{R_{\mathrm{fp}}}{z}\right)^{2 / s}\right]
\end{aligned}
$$

From these, we obtain the following expressions for the poloidal and total field strength along a field line:

$$
\begin{aligned}
B_{p}^{2}\left(\frac{z}{R_{\mathrm{fp}}}\right) & \approx\left(\frac{R_{\mathrm{fp}}}{z}\right)^{4(s-1) / s}\left[1-\left(\frac{R_{\mathrm{fp}}}{z}\right)^{2 / s}\right], \quad(\mathrm{A} 21) \\
B^{2}\left(\frac{z}{R_{\mathrm{fp}}}\right) & \approx M^{2}\left(\frac{R_{\mathrm{fp}}}{z}\right)^{2(s-1) / s} \quad(\mathrm{~A} 22) \\
& \times\left[1-\left(\frac{R_{\mathrm{fp}}}{z}\right)^{2 / s}+\frac{1}{M^{2}}\left(\frac{R_{\mathrm{fp}}}{z}\right)^{2(s-1) / s}\right] .
\end{aligned}
$$

Substituting these expressions and (A14) in equation (36), we obtain the following estimate for the minimum Lorentz factor along a field line,

$\gamma_{\min } \approx\left[\left(\frac{R_{\mathrm{fp}}}{z}\right)^{2 / s}+\frac{1}{M^{2}}\left(\frac{R_{\mathrm{fp}}}{z}\right)^{2(s-1) / s}\right]^{-1 / 2}$.

Since there are two terms, we need to keep track of the behaviour of each.

First, consider the case $\nu>1$. In this case, we saw earlier that $1 / s=\nu / 2>1 / 2$. Therefore, for large $z / R_{\mathrm{fp}}$, the second term in equation A23 always dominates, and so we have

$\gamma_{\min } \approx M\left(\frac{z}{R_{\mathrm{fp}}}\right)^{(s-1) / s}=M\left(\frac{z}{R_{\mathrm{fp}}}\right)^{(2-\nu) / 2}, \quad \nu>1$,

where we have substituted for $s$ using equation (A7). For $\nu=5 / 4$, this gives $\gamma_{\min } \propto z^{3 / 8}$, which agrees very well with the scaling we find for the numerical solution shown in Figure 4 (thick solid line).

Consider next $\nu<1$. In this case, the second term in equation A23 dominates for a range of $z / R_{\mathrm{fp}}$ up to a certain limit and the first term dominates beyond that, i.e.,

$\gamma_{\min } \approx M\left(\frac{z}{R_{\mathrm{fp}}}\right)^{(s-1) / s}, \quad \nu<1, \quad z<z_{\text {crit }},(\mathrm{A} 25)$ 
$\gamma_{\min } \approx\left(\frac{z}{R_{\mathrm{fp}}}\right)^{1 / s}, \quad \nu<1, \quad z>z_{\mathrm{crit}}$,

where the transition value of $z$ is given by

$z_{\text {crit }}=R_{\mathrm{fp}} M^{-s /(s-2)}$.

The two regimes of $\gamma_{\min }$ are seen clearly in the bottom right panel of Figure 9.

Before concluding, we remind the reader once again that all the relations given in this subsection have the correct scalings, but we have ignored numerical coefficients. For instance, the coefficient $M$ in the expressions for $B_{\phi}$ and $\gamma_{\min }$ is perhaps more appropriately written as $s M$. However, $s$ is a quantity of order unity and so, in the general spirit of neglecting coefficients, we have ignored this refinement. The other point is that we ignored line 2 of the differential equation A1 in the discussion here, whereas the terms in this line are negligible only for a limited range of $s$. A more complete analysis would retain these terms, but this is beyond the scope of the paper.

\section{REFERENCES}

Appl, S., \& Camenzind, M. 1992, A\&A, 256, 354

Appl, S., \& Camenzind, M. 1993, A\&A, 274, 699

Beskin, V. S. 1997, Uspekhi Fizicheskikh Nauk, 40, 659

Beskin, V. S., \& Malyshkin, L. M. 2000, Astron. Lett., 26, 208

Beskin, V. S., \& Nokhrino, E. E. 2006, MNRAS, 367, 375

Beskin, V. S., \& Pariev, V. N. 1993, Phys. Uspekhi, 36, 529

Blandford, R. D. 1976, MNRAS, 176, 465

Blandford, R. D., \& Payne, D. G. 1982, MNRAS, 199, 883

Blandford, R. D, \& Znajek, R. L. 1977, MNRAS, 179, 433

Bogovalov, S. V. 1997, A\&A, 323, 634

Contopoulos, J. 1994, ApJ, 432, 508

Contopoulos, J., \& Lovelace, R. V. E. 1994, ApJ, 429, 139

Contopoulos, J. 1995a, ApJ, 446, 67

Contopoulos, J. 1995b, ApJ, 450, 616

Gammie, C. F., McKinney, J. C., \& Tóth, G. 2003, ApJ, 589,444

Gammie, C. F., Shapiro, S. L., \& McKinney, J. C. 2004, ApJ, 602, 312

Goldreich, P., \& Julian, W. H. 1969, ApJ, 157, 869

Goldreich, P., \& Julian, W. H. 1970, ApJ, 160, 971

Heyvaerts, J., \& Norman, J. 1989, ApJ, 347, 1055

Kennel, C., Fujimura, F., \& Okamoto, I. 1983, Geophys. Astrophys. Fluid Dyn., 26, 147

Komissarov, S. S. 2001, MNRAS, 326, L41

Komissarov, S. S. 2002a, ArXiv Astrophysics e-prints, arXiv:astro-ph/0211141

Komissarov, S. S. 2002b, MNRAS, 336, 759

Komissarov, S. S. 2004, MNRAS, 350, 427

Komissarov, S. S. 2006, MNRAS, 367, 19

Li, Z.-Y., Chiueh, T., \& Begelman, M. C. 1992, ApJ, 394, 459

Lovelace, R. V. E. 1976, Nature, 262, 649

Lovelace, R. V. E., Mehanian, C., Mobarry, C. M., \& Sulka-

nen, M. E. 1986, ApJS, 62, 1

McKinney, J. C. 2005, ApJ, 630, L5

McKinney, J. C. 2006a, MNRAS, 367, 1797

McKinney, J. C. 2006b, MNRAS, 368, L30

McKinney, J. C. 2006c, MNRAS, 368, 1561
McKinney, J. C., \& Gammie, C. F. 2004, ApJ, 611, 977

McKinney, J. C. \& Narayan, R. 2006a, MNRAS, submitted (astro-ph/0607575)

McKinney, J. C. \& Narayan, R. 2006b, MNRAS, accepted (astro-ph/0607576)

Mestel, L. 1973, Astrophys. Space Sci., 24, 289

Michel, F. C. 1969, ApJ, 158, 727

Michel, F. C. 1973a, ApJ, 180, L133

Michel, F. C. 1973b, ApJ, 180, 207

Narayan, R., \& Yi, I. 1994, ApJ, 428, L13

Narayan, R. \& Yi, I. 1995a, ApJ, 452, 710

Narayan, R., \& Yi, I. 1995b, ApJ, 444, 231

Nitta, S., Takahashi, M., \& Tomimatsu, A. 1991. Phys. Rev. D, 44, 2295

Okamoto, I. 1974, MNRAS, 167, 457

Okamoto, I. 1978, MNRAS, 185, 69

Okamoto, I. 1999, MNRAS, 307, 253

Okamoto, I. 2000, MNRAS, 318, 250

Ostriker, E. C. 1997, ApJ, 486, 291

Penrose, R. 1969, Rev. Nuo Cim., 1, 252

Scharlemann, E. T., \& Wagoner, R. V. 1973, ApJ, 182, 951

Spitkovsky, A. 2006, ApJ, 648, L51

Tomimatsu, A. 1994, PASJ, 46, 123

Tomimatsu, A., Matsuoka, T., \& Takahashi, M. 2001, Phys. Rev. D, 64, 123003

Tomimatsu, A., \& Takahashi, M. 2003, ApJ, 592, 321

Vlahakis, N. 2004, Ap\&SS, 293, 67

Vlahakis, N., \& Konigl, A. 2003, ApJ, 596, 1080 